\title{
Chaotic and Nonchaotic Behavior in Three-Dimensional Quadratic Systems: 5-1 Dissipative Cases
}

\author{
$\dagger F u$ Zhang and $\ddagger$ Jack Heidel \\ $\dagger$ Department of Mathematics and Computer Science, \\ Cheyney University of Pennsylvania, Cheyney, PA 19319,USA \\ $\ddagger$ Department of Mathematics, \\ University of Nebraska at Omaha, Omaha, NE 68182,USA
}

\begin{abstract}
We show analytically that almost all three-dimensional dissipative quadratic systems of ordinary differential equations with a total of five terms on the right-hand side and one nonlinear term (namely 5-1 cases) are not chaotic except twenty one of them. Indeed we find nine systems that exhibit chaos, which were discovered by Sprott and Malasoma earlier. They are the simplest dissipative chaotic systems found so far. In this paper we also extend Heidel-Zhang's theorem which provides sufficient conditions for solutions in the three-dimensional autonomous systems with polynomials and rational expressions on the right-hand side being nonchaotic. We then investigate the twenty one systems analytically and numerically. We show the portraits of some typical chaotic and non-chaotic solutions in phase space. For two of the systems that exhibit chaos we found stable period 1, 2, 4, 8 and 12 orbits numerically.
\end{abstract}

\section{Introduction}

In this paper we study the chaotic and nonchaotic behavior of the set of all three-dimensional dissipative quadratic systems with a total of five terms on the right-hand side and one nonlinear term. The Lorenz equation [7] and Rössler system [9] both with seven terms on the right-hand side do exhibit chaos for certain parameter values. Sprott studied quadratic systems with less than seven terms and more than four terms on the right-hand side in [10] and found numerous cases of chaos in systems with six terms on the right-hand side and only one nonlinear (quadratic) term and systems with five terms and two nonlinear terms. In [12] and [13] Sprott also found examples of chaotic systems with five terms and only one nonlinear term.

In [14] Zhang and Heidel proved that all of the three-dimensional dissipative quadratic systems with a total of four terms on the right-hand side are not chaotic. Later in [5] Heidel and Zhang proved that almost all of the three-dimensional conservative quadratic systems with four terms on the right-hand side are not chaotic. The only system that is not proved being non-chaotic completely is $x^{\prime}=y^{2}-z^{2}, y^{\prime}=x, z^{\prime}=y$. Numerical studies show that this system does not exhibit chaos. More recently Heidel and Zhang ([6], 2007) made much progress on determining if a system is nonchaotic. The authors proved a theorem which provides sufficient conditions for solutions in three-dimensional autonomous systems with polynomials and rational expressions on the right-hand side being nonchaotic. In [6] all three-dimensional conservative quadratic system with five terms on the right-hand side with one nonlinear term were studied and we showed analytically that all but five of them are nonchaotic. Our numerical simulations seem to show that only one of the five systems $x^{\prime}=y^{2}-z+A, y^{\prime}=z, z^{\prime}=x$, discovered by Sprott in [11], exhibit chaos.

In this paper we first change the second hypotheses $(H 2)$ of Theorem 2.1 in [6](2007) to include the torus and surfaces with more complex topology. Then we focus on the set of all three-dimensional 
dissipative systems with five terms and one quadratic term on the right-hand side (namely 5-1 cases). Our goal is to determine which systems exhibit chaos and show analytically that all the rest are nonchaotic.

According to our classification in [6] we start from all possible dissipative systems. After eliminating solvable systems, systems that are equivalent to two-dimensional systems, four-term systems, linear systems, and/or some linearly equivalent systems, we only have to consider 65 systems.

Each of the systems in this paper is rescaled as in the following example. The system

$$
\left\{\begin{array}{l}
\dot{x}=a y^{2}+b x+c z \\
\dot{y}=r x \\
\dot{z}=p y
\end{array} \cdot=\frac{d}{d \tau}\right.
$$

where $a, b, c, r, p \neq 0$ are constants, can be transformed into one of the following two systems

$$
\left\{\begin{array}{l}
X^{\prime}=Y^{2} \pm X+A Z \quad,=\frac{d}{d t} \\
Y^{\prime}=X \\
Z^{\prime}=Y
\end{array}\right.
$$

by the scalar transformation

$$
x=\alpha X, \quad y=\beta Y, \quad z=\gamma Z, \quad \tau=\delta t, \quad \delta>0
$$

for two different sets of parameters $a, b, c, r$, and $p$. More specifically

$$
\alpha=-\frac{b^{3}}{a r^{2}}, \quad \beta=\frac{b^{2}}{a r}, \quad \gamma=-\frac{b p}{a r}, \quad \delta=-\frac{1}{b}, \quad b<0,
$$

and system (1.1) is transformed into the " - " system;

$$
\alpha=\frac{b^{3}}{a r^{2}}, \quad \beta=\frac{b^{2}}{a r}, \quad \gamma=\frac{b p}{a r}, \quad \delta=\frac{1}{b}, \quad b>0,
$$

and system (1.1) is transformed into the "+" system. The "+" system and the " - " system are related by the following transformation

$$
t \mapsto-t, \quad X \mapsto-X, \quad Y \mapsto Y, \quad Z \mapsto-Z
$$

Studying the " - " system when $t \rightarrow \infty$ is the same as studying a system linearly equivalent to the "+" system when $t \rightarrow-\infty$ and vice versa. So it is sufficient to study the two systems both when $t \rightarrow \infty$. For convenience, we change the capital letters back to the small letters.

For systems with more terms on the right-hand side, after the above scalar transformation more parameters have to be kept. For expamle for systems with 6 terms, two parameters have to be kept.

In section 2 we prove Theorem 2.3 and give a supporting example. In section 3, we list all the threedimensional 5-1 dissipative systems without constant terms and prove analytically that 30 of the 44 systems are not chaotic. The remaining 14 systems are considered further in sections 5 and 7 . Section 4 gives a complete list of all three-dimensional 5-1 dissipative systems with constant terms and we prove that 14 of the 21 systems are not chaotic. The remaining 7 systems are studied further in section 5 and 7 . In section 6 we investigate the nine 5-1 dissipative systems that exhibit chaos. 


\section{Nonchoatic Behavior on torus and surfaces with more complex topology}

In [6] we considered the autonomous system

$$
x^{\prime}=f(x), \quad x \in \mathbb{R}^{N}, \quad t \in \mathbb{R}
$$

where ${ }^{\prime}=\frac{d}{d t}, f$ is a map from $D \subseteq \mathbb{R}^{N}$ to $\mathbb{R}^{N}$. Let $x(0)=x_{0}$, and $x_{j}, x_{0 j}$ and $f_{j}, j=1,2, \ldots, N$ be the $j$ th component of $x, x_{0}$ and $f$, respectively. $f_{j}$ s are polynomials or rational expressions with $\mathrm{N}$ spatial variables. In the hypotheses of Theorem 2.1 in [6] the authors assumed simpler topology on simple surfaces. But torus or more complex topology are not considered. In this paper the authors extend the hypotheses in Theorem 2.1 in [6] to that each simple surface is homeomorphic to a two dimensional surface with any topology and so torus is certainly among them.

We will inherit the terminologies bounded and unbounded oscillatory, bounded chaos, isolated fixed point, simple surface, complex surface, isolated surface etc. defined in [6].

Next we recall some concepts in algebra. Let $P(x)=\Sigma_{\alpha} A_{\alpha} x^{\alpha}$ be a polynomial, where $x \in \mathbb{R}^{N}, N \geqslant 1$ is an integer, $\alpha=\left(\alpha_{1}, \ldots, \alpha_{N}\right)$, and each of the $\alpha_{i}$ 's is a nonnegative integer, $x^{\alpha}=x_{1}^{\alpha_{1}} \ldots x_{N}^{\alpha_{N}}$, the order of the multi-index $\alpha$ is denoted by $|\alpha|=\alpha_{1}+\ldots+\alpha_{N}$ and $A_{\alpha} \in \mathbb{R}$. The maximum order of all terms is the degree of the polynomial. An $n$th degree irreducible polynomial is a polynomial that can not be expressed as the product of two polynomials both with degree less than $n$. By the fundamental theorem of algebra, every polynomial in the above form can be factored as the product of irreducible polynomials with real coefficients. Therefore for some $m \leqslant \max |\alpha|$

$$
P(x)=\prod_{j=1}^{m} P_{j}(x),
$$

where $P_{j}(x)$ 's are irreducible polynomials and the zero set $\left\{x \in \mathbb{R}^{N} \mid P(x)=0\right\}$ of $P(x)$ is the union of the zero sets of $P_{j}(x), j=1, \ldots, m$. The zero set of each of the $P_{j}(x)$ consists of a finite number of connected components and each of them has dimension at most $N-1$ in $\mathbb{R}^{N}$.

The following well known result will be applied in the proof of Theorem 2.3.

Lemma 2.1 Let $P_{1}(x)$ and $P_{2}(x), x \in \mathbb{R}^{3}$, be two irreducible polynomials and $P_{1}$ and $P_{2}$ are not proportional. Then the set $\left\{x \in \mathbb{R}^{3} \mid P_{1}(x)=0\right.$ and $\left.P_{2}(x)=0\right\}$ is either empty or has dimension zero or one.

Lemma 2.2 Let $P(x), x \in \mathbb{R}^{3}$ be a polynomial. Then there exist two polynomials $P_{1}(x)$ and $P_{2}(x)$ such that $P(x)=P_{1}(x) P_{2}(x)$, and the zero set of each irreducible polynomial factor of $P_{1}(x)$ is nonempty and the zero set of $P_{2}(x)$ is empty.

Now we make the following assumptions on system (2.1) for $N=3$ :

$(H 1)$ System (2.1) is equivalent to

$$
F^{+}\left(x_{j}^{\prime \prime}, x_{j}^{\prime}, x_{j}\right)=C^{+}+\int_{0}^{t} G^{+}\left(x_{j}^{\prime \prime}(s), x_{j}^{\prime}(s), x_{j}(s)\right) d s, \quad,=\frac{d}{d t}
$$

or

$$
F^{+}(x, y, z)=C^{+}+\int_{0}^{t} G^{+}(x(s), y(s), z(s)) d s, \quad '=\frac{d}{d t}
$$

for some integer $1 \leqslant j \leqslant 3$ and equivalent to

$$
F^{-}\left(\ddot{x}_{j}, \dot{x}_{j}, x_{j}\right)=C^{-}+\int_{0}^{\tau^{*}} G^{-}\left(\ddot{x}_{j}(s), \dot{x}_{j}(s), x_{j}(s)\right) d s, \quad \cdot=\frac{d}{d \tau}, \tau=-t
$$


or

$$
F^{-}(x, y, z)=C^{-}+\int_{0}^{\tau^{*}} G^{-}(x(s), y(s), z(s)) d s, \quad \cdot=\frac{d}{d \tau}, \tau=-t
$$

for some integer $1 \leqslant j \leqslant 3$, where $C^{ \pm}$are constants and $G^{ \pm}(y) \geqslant 0$ for all $y \in \mathbb{R}^{3}$.

(H2) Each of the functions $f(y)=\frac{r(y)}{q(y)}$ in $(2.1), F^{ \pm}(y)=\frac{R^{ \pm}(y)}{Q^{ \pm}(y)}$ and $G^{ \pm}(y)=\frac{R_{0}^{ \pm}(y)}{Q_{0}^{ \pm}(y)}$ in (2.3) and (2.5), $y \in \mathbb{R}^{3}$ is either a polynomial or a rational expression with $\min _{y \in \mathbb{R}^{3}}\left\{\left|Q^{ \pm}(y)\right|,\left|Q_{0}^{ \pm}(y)\right|\right\}>q_{0}>0$, where $r(y), q(y), R^{ \pm}(y), Q^{ \pm}(y), R_{0}^{ \pm}(y)$ and $Q_{0}^{ \pm}(y)$ are polynomials and $q_{0}$ is a constant. The intersection of $\left\{x \in \mathbb{R}^{3} \mid G^{ \pm}(x)=0\right\}$ and $\left\{x \in \mathbb{R}^{3} \mid \nabla G^{ \pm}(x) \cdot f(x)=0\right\}$ has dimension at most one.

Theorem 2.3 Under hypotheses (H1) and (H2) system (2.1) has no bounded chaos.

Proof of Theorem 2.3: Chaotic behavior in system (2.1) can happen as $t \rightarrow \infty$ and $t \rightarrow-\infty$. We show that if (2.3) holds, then the system is not chaotic when $t \rightarrow \infty$ and similarly if (2.5) holds, then the system is not chaotic when $t \rightarrow-\infty$. The proof is similar if we replace (2.3) and (2.5) by (2.4) and (2.6) respectively. Since the proof for the case when $t \rightarrow-\infty$ is the same as that when $t \rightarrow \infty$, it is sufficient to prove the case for $t \rightarrow \infty$. Without loss of generality we consider solutions for $t \geqslant 0$. For simplicity we denote $F^{+}$as $F$ and $G^{+}$as $G$ respectively. Then $(H 1)$ implies

$$
\frac{d}{d t} F\left(x_{j}^{\prime \prime}(t), x_{j}^{\prime}(t), x_{j}(t)\right)=G\left(x_{j}^{\prime \prime}(t), x_{j}^{\prime}(t), x_{j}(t)\right) \geqslant 0 .
$$

Therefore either $F\left(x_{j}^{\prime \prime}, x_{j}^{\prime}, x_{j}\right)$ has a limit $L>-\infty$, as $t \rightarrow \infty$, where

$$
L=C+\int_{0}^{\infty} G\left(x_{j}^{\prime \prime}(s), x_{j}^{\prime}(s), x_{j}(s)\right) d s
$$

or $F\left(x_{j}^{\prime \prime}, x_{j}^{\prime}, x_{j}\right) \rightarrow \infty$, as $t \rightarrow \omega<\infty$. For the latter case, (H2) implies that at least one of the $x_{j}$, $j=1,2,3$ is unbounded and therefore the solutions are not chaotic when $t$ increases. For the first case we will show that the solutions are not chaotic by checking all the possible behaviors of the system. We define the following two sets of the initial conditions $x(0)$ and consider the solutions in the two sets separately.

$$
\begin{gathered}
\Omega_{1}=\left\{\left(x(0) \in \mathbb{R}^{3} \mid F\left(x_{j}^{\prime \prime}(t), x_{j}^{\prime}(t), x_{j}(t)\right)=\infty, \text { as } t \rightarrow \infty\right\},\right. \\
\Omega_{2}=\left\{\left(x(0) \in \mathbb{R}^{3} \mid F\left(x_{j}^{\prime \prime}(t), x_{j}^{\prime}(t), x_{j}(t)\right)=L<\infty \text { as } t \rightarrow \infty\right\}\right.
\end{gathered}
$$

Case 1. $x(0) \in \Omega_{1}$

Then $(H 2)$ implies again that at least one of the $x_{j}, j=1,2,3$ is unbounded and therefore the solutions are not chaotic when $t \rightarrow \infty$.

Case 2. $x(0) \in \Omega_{2}$.

Then $\left.\int_{0}^{\infty} G^{(} x_{j}^{\prime \prime}(s), x_{j}^{\prime}(s), x_{j}(s)\right) d s$ is finite. We consider $G \nrightarrow 0$ and $G \rightarrow 0$ as $t \rightarrow \infty$ in the following two sub cases.

Case $2.1 G \nrightarrow 0$ as $t \rightarrow \infty$.

Since $\int_{0}^{\infty} G d s$ is bounded, $G^{\prime}$ is unbounded. (H2) and the fact $G^{\prime}$ is unbounded implies that at least one of the $x_{j}, j=1,2,3$ is unbounded. Therefore the solutions are not chaotic. For convenience, we define the set $\Omega_{2}^{\dagger}$ as

$$
\Omega_{2}^{\dagger}=\left\{x(0) \in \Omega_{2}: \lim _{t \rightarrow \infty} G=0\right\} .
$$

Case $2.2 x(0) \in \Omega_{2}^{\dagger}$, i.e. $G \rightarrow 0$, as $t \rightarrow \infty$.

Then we have:

$$
F\left(x_{j}^{\prime \prime}(t), x_{j}^{\prime}(t), x_{j}(t)\right) \rightarrow L \text { and } G\left(x_{j}^{\prime \prime}(t), x_{j}^{\prime}(t), x_{j}(t)\right) \rightarrow 0 \text { as } t \rightarrow \infty
$$


Now we define the following two sets:

$S_{1}:=\left\{x \in \mathbb{R}^{3} \mid \tilde{F}(x)=F\left(x_{j}^{\prime \prime}, x_{j}^{\prime}, x_{j}\right)-L=0\right\}$ and $S_{2}:=\left\{x \in \mathbb{R}^{3} \mid \tilde{G}(x)=G\left(x_{j}^{\prime \prime}, x_{j}^{\prime}, x_{j}\right)=0\right\}$

Then by $(H 2) \tilde{F}(x)$ and $\tilde{G}(x)$ are polynomials or rational expressions in $x$. Here we recall the distance of two sets $\theta_{1}$ and $\theta_{2}$ in $\mathbb{R}^{3}$

$$
d\left(\theta_{1}, \theta_{2}\right)=\inf _{x \in \theta_{1}, y \in \theta_{2}}\|x-y\|
$$

where $\|\cdot\|$ denotes the Euclidean norm. From (2.7) for any solution $x$ with $x(0) \in \Omega_{2}^{\dagger}, d\left(x, S_{1}\right) \rightarrow 0$ and $d\left(x, S_{2}\right) \rightarrow 0$, as $t \rightarrow \infty$. Since $S_{1}$ and $S_{2}$ are close sets, $S_{1} \cap S_{2}$ is not empty and the solutions asymptote to an $\omega$-limit set $\Omega_{\omega} \subset S_{1} \cap S_{2}$.

Since $\tilde{F}(x)$ and $\tilde{G}(x)$ are polynomials or rational expressions, the connected components of $S_{1} \cap S_{2}$ is a collection of a finite number of points, a finite number of one dimensional curves closed or not closed, a finite number of disjoint two dimensional surfaces and a finite number of unions of one dimensional curves and two dimensional surfaces in $\mathbb{R}^{3}$. Let $n$ be the total number of connected components in $S_{1} \cap S_{2}$ and denote each of the components $I_{i}, i=1, \ldots, n$. We again classify the initial conditions $x(0) \in \Omega_{2}^{\dagger}$ as:

$$
\Omega_{2.1}^{\dagger}=\left\{x(0) \in \Omega_{2}^{\dagger}: \lim _{t \rightarrow \infty} d\left(x(t), I_{i}\right)=0, \operatorname{dim}\left\{I_{i}\right\}=0 / 1 \text { for some } i=1, . ., n .\right\}
$$

and

$$
\Omega_{2.2}^{\dagger}=\left\{x(0) \in \Omega_{2}^{\dagger}: \lim _{t \rightarrow \infty} d\left(x(t), I_{i}\right)=0, \operatorname{dim}\left\{I_{i}\right\}=2 \text { for some } i=1, . ., n .\right\} .
$$

Then $\Omega_{2.1}^{\dagger} \cup \Omega_{2.2}^{\dagger}=\Omega_{2}^{\dagger}$ and $\Omega_{2.1}^{\dagger} \cap \Omega_{2.2}^{\dagger}=\phi$.

Case $2.2 .1 x(0) \in \Omega_{2.1}^{\dagger}$.

(H2) implies that the one dimensional curves are intersections of the surfaces of irreducible polynomials. Therefore each solution in this case can only either approaches an equilibrium, or a limit cycle or goes to infinity. Therefore the solution can not be chaotic.

Case $2.2 .2 x(0) \in \Omega_{2.2}^{\dagger}$.

Without loss of generality, we consider when $\tilde{F}(x)$ and $\tilde{G}(x)$ are polynomials. Then by the fundamental theorem of algebra,

$$
\tilde{F}(x)=\prod_{\tilde{j}=1}^{m_{1}} \tilde{F}_{\tilde{j}}(x) \text { and } \tilde{G}(x)=\prod_{j=1}^{m_{2}} \tilde{G}_{j}(x)
$$

where $\tilde{F}_{\tilde{j}}(x)$ 's and $\tilde{G}_{j}(x)$ 's are irreducible polynomials. Note that in this case by $(H 2)$ and lemma 2.1 there exists an $l$ such that $\tilde{F}_{\tilde{j}_{1}}(x) \equiv \tilde{G}_{j_{1}}(x), \ldots, \tilde{F}_{\tilde{j}_{l}}(x) \equiv \tilde{G}_{j_{l}}(x)$, for all $x \in \mathbb{R}^{3}$.

We call the set $\left\{x \in \mathbb{R}^{3} \mid M(x)=0\right\}$ a positively invariant set under the flow $x^{\prime}=f(x)$ if $M(x(0))=0$ implies $M(x(t))=0$ for all $t>0$. Then obviously if $\left.(\nabla M, f)\right|_{M=0} \equiv 0, M=0$ is positively invariant under the flow.

Here for each of the $I_{i}$ with dimension 2, we define

$$
d_{i}=\max _{k=1, . ., l_{i}}\left\{\operatorname{dim}\left\{x \in \mathbb{R}^{3} \mid \nabla \tilde{F}_{\tilde{j}_{k}}(x) \cdot f=0\right\}\right\}
$$

and

$$
\Omega_{2.2 .1}^{\dagger}=\left\{x(0) \in \Omega_{2.2}^{\dagger}: \lim _{t \rightarrow \infty} d\left(x(t), I_{i}\right)=0, d_{i} \leqslant 1 \text { for some } i=1, . ., n .\right\}
$$

and by $(H 2)$

$$
\Omega_{2.2 .2}^{\dagger}=\left\{x(0) \in \Omega_{2.2}^{\dagger}: \lim _{t \rightarrow \infty} d\left(x(t), I_{i}\right)=0, d_{i}=2 \text { for some } i=1, . ., n .\right\}=\phi,
$$

where $\phi$ is the empty set.

Hence $x(0) \in \Omega_{2.2 .1}^{\dagger} \Leftrightarrow x(0) \in \Omega_{2.2}^{\dagger}$. 
Clearly each of the solutions in this case will either asymptote to a curve which is an intersection of the zero sets of two irreducible polynomials or an equilibrium. Therefore they are not chaotic.

This completes the proof of the theorem.

In the following example we will see that there is an invariant manifold on a two dimensional torus. We will apply our theorem to show that the system is not chaotic.

$$
\left\{\begin{array}{l}
x^{\prime}=y \\
y^{\prime}=z \\
z^{\prime}=\frac{4 R^{2}(2 x y+2 y z)\left( \pm y^{3}+A\right)-(4 x y+4 y z)\left( \pm y^{3}+A\right) \Theta \mp 3 y^{2} z T \pm T^{2} x^{2}}{4 z\left( \pm y^{3}+A\right) \Theta}
\end{array}\right.
$$

where $T(x, y, z)=\left(x^{2}+y^{2}+z^{2}+R^{2}-r^{2}\right)^{2}-4 R^{2}\left(x^{2}+y^{2}\right)=\Theta^{2}-4 R^{2}\left(x^{2}+y^{2}\right)$, $\Theta=x^{2}+y^{2}+z^{2}+R^{2}-r^{2}$, where $R>r>0$, and $A$ are constants.

After this scalar transformations $x=X, y=-Y, z=Z, t=-\tau$, the " + " system of (2.8) is transformed to the " - " system.

Proof: For "+" system of (2.8), we have

$$
\begin{gathered}
4 z\left(y^{3}+A\right) \Theta z^{\prime}=4 R^{2}(2 x y+2 y z)\left(y^{3}+A\right)-(4 x y+4 y z)\left(y^{3}+A\right) \Theta-3 y^{2} z T+T^{2} x^{2} \\
\left\{\left(4 x x^{\prime}+4 y y^{\prime}\right) \Theta+4 z z^{\prime} \Theta-4 R^{2}\left(2 x x^{\prime}+2 y y^{\prime}\right)\right\}\left(y^{3}+A\right)+3 y^{2} y^{\prime} T=T^{2} x^{2}
\end{gathered}
$$

Since $T(x, y, z)=\left(x^{2}+y^{2}+z^{2}+R^{2}-r^{2}\right)^{2}-4 R^{2}\left(x^{2}+y^{2}\right)=\Theta^{2}-4 R^{2}\left(x^{2}+y^{2}\right)$,

$$
T^{\prime}=\left(4 x x^{\prime}+4 y y^{\prime}+4 z z^{\prime}\right) \Theta-4 R^{2}\left(2 x x^{\prime}+2 y y^{\prime}\right)
$$

We have

$$
T^{\prime}\left(y^{3}+A\right)+3 y^{2} y^{\prime} T=T^{2} x^{2}
$$

Integrate to get

$$
T\left(y^{3}+A\right)=C+\int_{0}^{t} T^{2} x^{2} d s
$$

where $C$ is a constant. Since the zero set of $T$ is a torus, we need to check $(H 2)$ of the theorem. We first calculate the gradient of $T$

$$
\nabla T=\left(4 x \Theta-4 R^{2} x, 4 y \Theta-4 R^{2} y, 4 z \Theta\right)
$$

and then calculate the dot product of $\nabla T$ and the vector field

$$
\begin{gathered}
(\nabla T) \cdot\left(x^{\prime}, y^{\prime}, z^{\prime}\right)=0 \\
\left\{\begin{array}{c}
(\nabla T) \cdot\left(x^{\prime}, y^{\prime}, z^{\prime}\right)=\left(4 x \Theta-4 R^{2} x\right) y+\left(4 y \Theta-4 R^{2} y\right) z+4 z \Theta z^{\prime}=0 \\
T=\left(x^{2}+y^{2}+z^{2}+R^{2}-r^{2}\right)^{2}-4 R^{2}\left(x^{2}+y^{2}\right)=0 \\
\text { where } z^{\prime}=\frac{4 R^{2}(2 x y+2 y z)\left(y^{3}+A\right)-(4 x y+4 y z)\left(y^{3}+A\right) \Theta-3 y^{2} z T+T^{2} x^{2}}{4 z\left(y^{3}+A\right) \Theta}
\end{array}\right.
\end{gathered}
$$

Obviously the solution to the above system of algebraic equations is an invariant manifold which has dimension one. Then by the above theorem, the "+" system is not chaotic.

For the " - " system of (2.8), we have

$$
T\left(y^{3}-A\right)=C+\int_{0}^{t} T^{2} x^{2} d s
$$

A similar argument to that of the (2.8)+ concludes that the " - " system is not chaotic.

For convenience in the next 2 sections, we let $\dot{x}=x^{\prime}=\frac{d x}{d t}$ and $t_{0}=0$. 
3 Five-term dissipative systems with one quadratic term and without constant terms.

Three-dimensional 5-1 dissipative systems without constant terms that need to be considered are the following 44 systems:

$$
\begin{aligned}
& \left\{\begin{array}{l}
x^{\prime}=y^{2} \pm x+A z \\
y^{\prime}=x \\
z^{\prime}=y
\end{array}\right. \\
& \left\{\begin{array}{l}
x^{\prime}=y^{2}+A x+z \\
y^{\prime}=x \\
z^{\prime}= \pm z
\end{array}\right. \\
& \left\{\begin{array}{l}
x^{\prime}=y^{2}+A y+z \\
y^{\prime}=x \\
z^{\prime}= \pm z
\end{array}\right. \\
& \left\{\begin{array}{l}
x^{\prime}=y^{2} \pm x+A y \\
y^{\prime}=z \\
z^{\prime}=x
\end{array}\right. \\
& \left\{\begin{array}{l}
x^{\prime}=y^{2} \pm x+A z \\
y^{\prime}=z \\
z^{\prime}=x
\end{array}\right. \\
& \left\{\begin{array}{l}
x^{\prime}=y z \pm x+A y \\
y^{\prime}=z \\
z^{\prime}=x
\end{array}\right. \\
& \left\{\begin{array}{l}
x^{\prime}= \pm x+y+A z \\
y^{\prime}=x^{2} \\
z^{\prime}=x
\end{array}\right. \\
& \left\{\begin{array}{l}
x^{\prime}= \pm x+y+A z \\
y^{\prime}=x^{2} \\
z^{\prime}=y
\end{array}\right. \\
& \left\{\begin{array}{l}
x^{\prime}=A x+y+z \\
y^{\prime}=x^{2} \\
z^{\prime}= \pm z
\end{array}\right. \\
& \left\{\begin{array}{l}
x^{\prime}= \pm x+y+A z \\
y^{\prime}=z^{2} \\
z^{\prime}=x
\end{array}\right. \\
& \left\{\begin{array}{l}
x^{\prime}= \pm x+y+A z \\
y^{\prime}=x z \\
z^{\prime}=y
\end{array}\right.
\end{aligned}
$$




$$
\begin{aligned}
& \left\{\begin{array}{l}
x^{\prime}=y^{2} \pm x \\
y^{\prime}=x+A z \\
z^{\prime}=x
\end{array}\right. \\
& \left\{\begin{array}{l}
x^{\prime}=y^{2} \pm x \\
y^{\prime}=x+A z \\
z^{\prime}=y
\end{array}\right. \\
& \left\{\begin{array}{l}
x^{\prime}=y^{2}+A x \\
y^{\prime}=x+z \\
z^{\prime}= \pm z
\end{array}\right. \\
& \left\{\begin{array}{l}
x^{\prime}=y^{2} \pm x \\
y^{\prime}=A y+z \\
z^{\prime}=x
\end{array}\right. \\
& \left\{\begin{array}{l}
x^{\prime}=y^{2}+A y \\
y^{\prime}=x+z \\
z^{\prime}= \pm z
\end{array}\right. \\
& \left\{\begin{array}{l}
x^{\prime}=y^{2}+A y \\
y^{\prime}= \pm y+z \\
z^{\prime}=x
\end{array}\right. \\
& \left\{\begin{array}{l}
x^{\prime}=y^{2}+A z \\
y^{\prime}=x \pm y \\
z^{\prime}=x
\end{array}\right. \\
& \left\{\begin{array}{l}
x^{\prime}=y^{2}+A z \\
y^{\prime}=x \pm y \\
z^{\prime}=y
\end{array}\right. \\
& \left\{\begin{array}{l}
x^{\prime}=y^{2}+z \\
y^{\prime}=x+A y \\
z^{\prime}= \pm z
\end{array}\right. \\
& \left\{\begin{array}{l}
x^{\prime}=y^{2}+A z \\
y^{\prime}=x+z \\
z^{\prime}= \pm z
\end{array}\right. \\
& \left\{\begin{array}{l}
x^{\prime}=y^{2}+A z \\
y^{\prime}= \pm y+z \\
z^{\prime}=x
\end{array}\right. \\
& \left\{\begin{array}{l}
x^{\prime}=z^{2}+A x \\
y^{\prime}=x \pm y \\
z^{\prime}=y
\end{array}\right.
\end{aligned}
$$




$$
\begin{aligned}
& \left\{\begin{array}{l}
x^{\prime}=z^{2} \pm x \\
y^{\prime}=x+A z \\
z^{\prime}=y
\end{array}\right. \\
& \left\{\begin{array}{l}
x^{\prime}=z^{2}+A y \\
y^{\prime}=x \pm y \\
z^{\prime}=x
\end{array}\right. \\
& \left\{\begin{array}{l}
x^{\prime}=z^{2}+A y \\
y^{\prime}=x \pm y \\
z^{\prime}=y
\end{array}\right. \\
& \left\{\begin{array}{l}
x^{\prime}=z^{2}+A y \\
y^{\prime}= \pm y+z \\
z^{\prime}=x
\end{array}\right. \\
& \left\{\begin{array}{l}
x^{\prime}=z^{2}+A z \\
y^{\prime}=x \pm y \\
z^{\prime}=y \\
y^{\prime}=x \pm y \\
z^{\prime}=x
\end{array}\right. \\
& \left\{\begin{array}{l}
x^{\prime}=y z+A x \\
x^{\prime}= \pm y+A \\
z^{\prime}=x \\
y^{\prime}=x
\end{array}\right. \\
& \left\{\begin{array}{l}
x^{\prime}=y z+A y \\
y^{\prime}=x \pm y \\
z^{\prime}=x \\
x^{\prime}=y z \pm x \\
z^{\prime}=x \\
y^{\prime}=x+A z
\end{array}\right. \\
& z^{\prime}=y
\end{aligned}
$$




$$
\left.\begin{array}{l}
\left\{\begin{array}{l}
x^{\prime}=y z+A z \\
y^{\prime}=x \pm y \\
z^{\prime}=y
\end{array}\right. \\
\left\{\begin{array}{l}
x^{\prime}=y z+A z \\
y^{\prime}= \pm y+z \\
z^{\prime}=x
\end{array}\right. \\
\left\{\begin{array}{l}
x^{\prime}= \pm x+A y \\
y^{\prime}=x+z \\
z^{\prime}=x^{2}
\end{array}\right. \\
\left\{\begin{array}{l}
x^{\prime}= \pm x+y \\
y^{\prime}=A y+z \\
z^{\prime}=x^{2}
\end{array}\right. \\
\left\{\begin{array}{l}
x^{\prime}= \pm x+A y \\
y^{\prime}=x+z \\
z^{\prime}=y^{2}
\end{array}\right. \\
\left\{\begin{array}{l}
x^{\prime}= \pm x+A z \\
y^{\prime}=x+z \\
z^{\prime}=y^{2}
\end{array}\right. \\
\left\{\begin{array}{l}
x^{\prime}= \pm x+A y \\
y^{\prime}=x+z \\
z^{\prime}=x y \\
y^{\prime}= \pm x+y \\
z^{\prime}=x y
\end{array}\right. \\
y^{\prime}= \pm x+z \\
z^{\prime}=x y
\end{array}\right]
$$

Among the 44 systems listed above 33 of them are dissipative when choosing " - " sign, except systems (3.2), (3.9), (3.14), (3.15), (3.20), (3.23), (3.29), (3.32), (3.39), (3.43) and (3.44) which are dissipative when $\pm 1+A<0$ by the definition of dissipative systems(see [6]). Although this paper focuses on dissipative systems in this section, it is convenient to deal with systems (3.2), (3.9), (3.14), (3.15), (3.20), (3.23), (3.29), (3.32), (3.39), (3.43) and (3.44) for both $\pm 1+A>0$ and $\pm 1+A<0$. We show in the next theorem that 30 of the 44 systems are not chaotic.

Theorem 3.1 Systems (3.2) $\backsim$ (3.5), (3.7) $\backsim$ (3.10), (3.12), (3.14) $\backsim$ (3.17), (3.20), (3.21), (3.23), $(3.25) \backsim(3.30),(3.32),(3.33)$, and $(3.38) \backsim(3.43)$ are not chaotic.

Proof. Systems (3.2), (3.3), (3.9), (3.14), (3.16), (3.20) and (3.21) all have that $z(t)=C e^{-t}$. Therefore $z \rightarrow 0$, as $t \rightarrow \infty$. Their scalar equations in another component are of the following forms: 


$$
\begin{array}{ll}
(3.2) & \ddot{y}-y^{2}-A \dot{y}=C e^{ \pm t} \\
(3.3) & \ddot{y}-y^{2}-A y=C e^{ \pm t} \\
(3.9) & \ddot{x}-x^{2}-A x= \pm C e^{ \pm t} \\
(3.14) & \ddot{y}-y^{2}-A \dot{y}=\left( \pm A-A^{2}\right) C e^{ \pm t} \\
(3.16) & \ddot{y}-y^{2}-A y= \pm C e^{ \pm t} \\
(3.20) & \ddot{y}-y^{2}=(A+1) C e^{ \pm t} \\
(3.21) & \ddot{y}-y^{2}=(A \pm 1) C e^{ \pm t}
\end{array}
$$$$
\text { (3.20) } \quad \ddot{y}-y^{2}=(A+1) C e^{ \pm t}
$$

where $A$ and $C$ are constants. For the equations with a " - " sign in (3.45) the RHSs approach zero as $t \rightarrow \infty$. By Theorem 2.1 in [6] the 7 systems are not chaotic. For the equations with a " + " sign in (3.45), the solutions of these equations are unbounded and therefore there is no bounded chaos in the 7 systems. Hence these 7 systems are nonchaotic.

Next we show that systems (3.4), (3.5), (3.8), (3.10), (3.12), (3.15), (3.17), (3.23), (3.25), (3.26), $(3.28) \backsim(3.30),(3.32),(3.38) \backsim(3.43)$ are not chaotic. The scalar equations and the integration of the scalar equations of these 20 systems are as follows:

$$
\begin{aligned}
(3.4) \pm: & \dddot{y}=y^{2} \pm \ddot{y}+A y, \quad \text { multiply by } \dot{y} \text { and integrate } \\
\pm: & \dot{y} \ddot{y}-\frac{1}{3} y^{3}-\frac{A}{2} y^{2} \mp \frac{1}{2} \dot{y}^{2}=C+\int_{0}^{t} \ddot{y}^{2}(s) d s \\
(3.5) \pm: & \dddot{y}=y^{2} \pm \ddot{y}+A \dot{y}, \quad \text { integrate } \\
\pm: & \ddot{y} \mp \dot{y}-A y=C+\int_{0}^{t} y^{2}(s) d s \\
(3.8) \pm: & \dddot{x}=A x^{2} \pm \ddot{x}+2 x \dot{x}, \quad \text { integrate } \\
\pm: & \ddot{x} \mp \dot{x}-x^{2}=C+\int_{0}^{t} A x^{2}(s) d s \\
(3.10) \pm: & \dddot{z}=z^{2} \pm \ddot{z}+A \dot{z}, \quad \text { integrate } \\
\pm: & \ddot{z} \mp \dot{z}-A z=C+\int_{0}^{t} z^{2}(s) d s \\
(3.12) \pm: & \dddot{y}=A y^{2} \pm \ddot{y}+2 y \dot{y}, \quad \text { integrate } \\
\pm: & \ddot{y} \mp \dot{y}-y^{2}=C+\int_{0}^{t} A y^{2}(s) d s \\
(3.15) \pm: & \dddot{y}=y^{2}+(A \pm 1) \ddot{y} \mp A \dot{y}, \quad \text { integrate } \\
\pm: & \ddot{y}-(A \pm 1) \dot{y} \pm y=C+\int_{0}^{t} y^{2}(s) d s \\
(3.17) \pm: & \dddot{y}=y^{2} \pm \ddot{y}+A y \quad \text { multiply by } \dot{y} \text { and integrate } \\
\pm: & \ddot{y}-\frac{1}{3} y^{3}-\frac{A}{2} y^{2} \mp \frac{1}{2} \dot{y}^{2}=C+\int_{0}^{t} \ddot{y}^{2}(s) d s \\
\pm: & \ddot{z}-(A \pm 1) \dot{z} \pm A z=C+\int_{0}^{t} z^{2}(s) d s \\
(3.23) \pm: & \dddot{z}=z^{2}+(A \pm 1) \ddot{z} \mp A \dot{z}, \quad \text { integrate } \\
\pm &
\end{aligned}
$$


$(3.25) \pm: \quad \dddot{z}=\mp z^{2} \pm \ddot{z}+A \dot{z}+2 z \dot{z}, \quad$ multiply by $\dot{z}$ integrate

$\pm: \quad \ddot{z} \mp \dot{z} \pm z^{2}-A z=C+\int_{0}^{t} z^{2}(s) d s$

$(3.26) \pm: \quad \dddot{z}=z^{2}+(A \pm 1) \dot{z}, \quad$ integrate

$\pm: \quad \ddot{z}-(A \pm 1) y=C+\int_{0}^{t} z^{2}(s) d s$

$(3.28) \pm: \quad \dddot{z}=z^{2} \pm \ddot{z}+A z, \quad$ integrate

$\pm: \quad \dot{z} \ddot{z}-\frac{1}{3} z^{3}-\frac{A}{2} z^{2} \mp \frac{1}{2} \dot{z}^{2}=C+\int_{0}^{t} \ddot{z}^{2}(s) d s$

$(3.32) \pm: \quad \dddot{y}=\mp y^{2} \pm \ddot{y}+y \dot{y}+A(\dot{y} \mp \dot{y}) \quad$ integrate

$\pm: \quad \ddot{y}-\frac{1}{2} y^{2}-A(y \pm y)=C \mp \int_{0}^{t} y^{2}(s) d s$

$(3.38) \pm: \quad \dddot{x}=A x^{2} \pm \ddot{x}+A \dot{x}, \quad$ integrate

$\pm: \quad \ddot{x} \mp \dot{x}-A x=C+A \int_{0}^{t} x^{2}(s) d s$

$(3.39) \pm: \quad \dddot{x}=x^{2}+(A \pm 1) \ddot{x} \mp A \dot{x}, \quad$ integrate

$\pm: \quad \ddot{x}-(A \pm 1) \dot{x} \pm A x=C+A \int_{0}^{t} x^{2}(s) d s$

$(3.40) \pm: \quad \dddot{y}=\mp y^{2}+(A \pm 1) \dot{y}+2 y \dot{y}, \quad$ integrate

$\pm: \quad \ddot{y}-(A \pm 1) \dot{y}-y^{2}=C \mp \int_{0}^{t} y^{2}(s) d s$

$(3.41) \pm: \quad \dddot{y}=(A \mp 1) y^{2} \pm \ddot{y}+2 y \dot{y}, \quad$ integrate

$\pm: \quad \ddot{y} \mp \dot{y}-y^{2}=C+(A \mp 1) \int_{0}^{t} y^{2}(s) d s$

$(3.42) \pm: \quad \dddot{x}=\mp x^{2} \pm \ddot{x}+A \dot{x}+x \dot{x}, \quad$ integrate

$\pm: \quad \ddot{x} \mp \dot{x}-A x-\frac{A}{2} x^{2}=C \mp \int_{0}^{t} x^{2}(s) d s$

$(3.43) \pm: \quad \dddot{x}=\mp x^{2}+(A \pm 1) \ddot{x} \mp A \dot{x}+x \dot{x}, \quad$ integrate

$\pm: \quad \ddot{x}-(A \pm 1) \dot{x} \pm A x-\frac{1}{2} x^{2}=C \mp \int_{0}^{t} x^{2}(s) d s$

where $C$ is a constant.

In system (3.29), let $u=y-z, u^{\prime}= \pm y$. Then its scalar equation is $u^{\prime \prime \prime} \mp(A+1) u^{\prime \prime}+u u^{\prime} \pm A u^{\prime}= \pm\left(u^{\prime}\right)^{2}$. Integrate to get

$$
u^{\prime \prime} \mp(A+1) u^{\prime}+\frac{1}{2} u^{2} \pm A u=C \pm \int_{0}^{t}\left(u^{\prime}(s)\right)^{2} d s
$$

where $C$ is a constant.

In system (3.30), let $u=y-z, u^{\prime}=A z$. Then its scalar equation is $u^{\prime \prime \prime} \mp u^{\prime \prime}-u u^{\prime}=\frac{1}{A}\left(u^{\prime}\right)^{2}$. Integrate to get

$$
u^{\prime \prime} \mp u^{\prime}-\frac{1}{2} u^{2}=C+\frac{1}{A} \int_{0}^{t}\left(u^{\prime}(s)\right)^{2} d s \quad A \neq 0
$$

where $C$ is a constant. 
When $A=0, z=y+B$ for some constant $B$, and therefore $x^{\prime}=y(y+B) \pm x, y^{\prime}=x$. By the Poincaré-Bendixon Theorem [4], the system is not chaotic. By Theorem 2.1 in [6], these 20 systems have no bounded chaos.

System (3.7) can be written as 2D scalar equation: $x^{\prime \prime}= \pm x^{\prime}+x^{2}+A x$. By Poincaré-Bendixon Theorem, the system is not chaotic.

Consider system (3.27), since $y^{\prime \prime}= \pm y^{\prime}+x, y^{\prime \prime \prime}= \pm y^{\prime \prime}+z^{2}+A y$ and $z=y^{\prime} \mp y$, we have

$$
\begin{gathered}
y^{\prime \prime \prime}= \pm y^{\prime \prime}+\left(y^{\prime} \mp y\right)^{2}+A y \quad \text { multiply by }\left(y^{\prime} \mp y\right)^{\prime}, \text { lead to } \\
y^{\prime \prime} y^{\prime \prime \prime} \mp y^{\prime} y^{\prime \prime \prime}= \pm\left(y^{\prime \prime}\right)^{2}-y^{\prime} y^{\prime \prime}+\left(y^{\prime} \mp y\right)^{2}\left(y^{\prime} \mp y\right)^{\prime}+A y y^{\prime \prime} \mp A y y^{\prime}
\end{gathered}
$$

integrate to get

$$
\left(y^{\prime \prime}\right)^{2} \mp 2 y^{\prime} y^{\prime \prime}+\left(y^{\prime}\right)^{2}-\frac{2}{3}\left(y^{\prime} \mp y\right)^{3} \pm A y^{2}-2 A y y^{\prime}=C-A \int_{0}^{t}\left(y^{\prime}(s)\right)^{2} d s
$$

where $C$ is a constant. By Theorem 2.1 in [6], the systems has no bounded chaos.

System (3.33) has a 1D invariant manifold $x=0, z=-A$ and $y=y_{0} e^{ \pm t}$, where $y_{0}$ is a constant. Its scalar equation reads

$$
(z+A) z^{\prime \prime \prime}=(z+A) z z^{\prime} \pm z z^{\prime \prime}+z^{\prime} z^{\prime \prime}+A(z+A) z^{\prime} \pm A z^{\prime \prime}
$$

Integrate to get

$$
z z^{\prime \prime}-z^{\prime 2}+A z^{\prime \prime}-\frac{1}{3} z^{3}-A z^{2} \mp z z^{\prime}-A^{2} z \mp A z^{\prime}=C+\int_{0}^{t}\left(z^{\prime}(s)\right)^{2} d s
$$

where $C$ is a constant. By Theorem 2.1 in [6], the system has no bounded chaos. This completes the proof of the theorem.

\section{Systems with constant terms}

All three-dimensional 5-1 dissipative systems with one quadratic term and constant terms that need to be considered are the following 21 systems:

$$
\begin{aligned}
& \left\{\begin{array}{l}
x^{\prime}=y^{2} \pm x+A \\
y^{\prime}=z \\
z^{\prime}=x
\end{array}\right. \\
& \left\{\begin{array}{l}
x^{\prime}=y^{2}+z+A \\
y^{\prime}=x \\
z^{\prime}= \pm z
\end{array}\right. \\
& \left\{\begin{array}{l}
x^{\prime}=y z \pm x+A \\
y^{\prime}=x \\
z^{\prime}=y
\end{array}\right. \\
& \left\{\begin{array}{l}
x^{\prime}=z^{2} \pm x+y \\
y^{\prime}=A \\
z^{\prime}=x
\end{array}\right.
\end{aligned}
$$




$$
\begin{aligned}
& \left\{\begin{array}{l}
\left\{\begin{array}{l}
x^{\prime}= \pm x+y+A \\
y^{\prime}=x z \\
z^{\prime}=y
\end{array}\right. \\
\left\{\begin{array}{l}
x^{\prime}= \pm x+z+A \\
y^{\prime}=x z \\
z^{\prime}=y
\end{array}\right. \\
\left\{\begin{array}{l}
x^{\prime}=y^{2}+A \\
y^{\prime}=x+z \\
z^{\prime}= \pm z
\end{array}\right. \\
\left\{\begin{array}{l}
x^{\prime}=y+A \\
y^{\prime}=z \pm y \\
z^{\prime}=x
\end{array}\right. \\
\left\{\begin{array}{l}
x^{\prime}=z^{2}+A \\
y^{\prime}=x \pm y \\
z^{\prime}=y
\end{array}\right. \\
\left\{\begin{array}{l}
x^{\prime}=y z \pm x \\
y^{\prime}=x+A \\
z^{\prime}=y
\end{array}\right. \\
\left\{\begin{array}{l}
x^{\prime}=y+A \\
y^{\prime}=x \pm y \\
z^{\prime}=x \\
z^{\prime}=x+A
\end{array}\right. \\
\left\{\begin{array}{l}
x^{\prime}=y z+A \\
y^{\prime}=x \pm y \\
z^{\prime}=y
\end{array}\right. \\
y^{\prime}= \pm y+A \\
z^{\prime}=x
\end{array}\right. \\
& y^{\prime}=x+A
\end{aligned}
$$




$$
\begin{aligned}
& \left\{\begin{array}{l}
x^{\prime}=y z \pm x \\
y^{\prime}=z+A \\
z^{\prime}=x
\end{array}\right. \\
& \left\{\begin{array}{l}
x^{\prime}=y^{2} \pm x \\
y^{\prime}=x+z \\
z^{\prime}=A
\end{array}\right. \\
& \left\{\begin{array}{l}
x^{\prime}=y^{2}+z \\
y^{\prime}=x \pm y \\
z^{\prime}=A
\end{array}\right. \\
& \left\{\begin{array}{l}
x^{\prime}= \pm x+z \\
y^{\prime}=x+A \\
z^{\prime}=x y
\end{array}\right. \\
& \left\{\begin{array}{l}
x^{\prime}= \pm x+z \\
y^{\prime}=z+A \\
z^{\prime}=x y
\end{array}\right.
\end{aligned}
$$

We refer the "+" systems as $(n a)$ and the " - " ones as $(n b)$, where the $n$ represents one of the labels $4.1,4.2, \ldots, 4.21$.

Theorem 4.1 Systems (4.1)-(4.4), (4.7)-(4.9), (4.11), (4.13)-(4.16), (4.18), and (4.19) are not chaotic.

Proposition 4.2 Systems (4.1), (4.2) and (4.3) are equivalent to (4.13), (4.14) and (4.16), respectively.

Proof. (1) For system (4.1), let $X=x \mp A, Y=z, Z=y$. Then system (4.1) is equivalent to (4.13). (2) For system (4.14), let $X=x, Y=y \mp A, Z=y$. Then system (4.14) is equivalent to (4.2). (3) Finally, for system (4.3), let $X=x \mp A, Y=y, Z=z$. Then system (4.3) is equivalent to (4.16).

Proof of Theorem 4.1. Systems (4.2), (4.3), (4.4), (4.7), (4.11), (4.15), (4.18), and (4.19) are transformed to the following equations respectively.:

$$
\begin{array}{ll}
(4.2) & \ddot{y}-y^{2}=C e^{ \pm t}+A, \quad z=C e^{ \pm t} \\
(4.3) & \ddot{z}-\frac{1}{2} z^{2} \mp \dot{z}=A t+C \\
(4.4) & \ddot{z}-z^{2} \mp \dot{z}=A t+C, \quad y=A t+C \\
(4.7) & \ddot{y}-y^{2}=C e^{ \pm t}+A, \quad z(t)=C e^{ \pm t} \\
(4.11) & \ddot{z}-\frac{1}{2} z^{2} \mp \dot{z}=A t+C \\
(4.15) & \ddot{z}-z^{2}-(A t+C) z \mp \dot{z}=0, \quad y-z=A t+C \\
(4.18) & \ddot{y}-y^{2} \mp \dot{y}=\mp A t \mp C+A, \quad z=A t+C \\
(4.19) & \ddot{y}-y^{2} \mp \dot{y}=A t+C, \quad z=A t+C
\end{array}
$$

where $C$ is a constant. Among the above systems (4.2) is the same as (4.7), (4.3) is the same as (4.11) which is equivalent to (4.4), (4.18) and (4.19). So it is sufficient to consider systems (4.2), (4.3), and (4.15). For the "+" systems, since the RHS of (4.2) and (4.3) both go to infinity, one of the three variables $x, y$ and $z$ of LHS in them is unbounded. For the " - " systems, since the RHS of (4.2) and (4.3) go to zero, 
by Theorem 2.1 in [6] the systems are not chaotic. From system (4.15) we have $y-z=A t+C$. Then the argument is the same as that of (4.2).

Since (4.1) is equivalent to (4.13), we only need to consider (4.1), (4.8), and (4.9). Their scalar equations and integration of the scalar equations are as follows:

$$
\begin{aligned}
(4.1) \pm: & \dddot{y}=y^{2} \pm \ddot{y}+A, \quad \text { multiply by } \dot{y} \text { and integrate } \\
\pm: & \dot{y} \ddot{y}-\frac{1}{3} y^{3} \mp \frac{1}{2} \dot{y}^{2}-A y=C+\int_{0}^{t} \ddot{y}^{2}(s) d s \\
(4.8) \pm: & \dddot{y}=y^{2} \pm \ddot{y}+A, \quad \text { multiply by } \dot{y} \text { and integrate } \\
\pm: & \dot{y} \ddot{y}-\frac{1}{3} y^{3} \mp \frac{1}{2} \dot{y}^{2}-A y=C+\int_{0}^{t} \ddot{y}^{2}(s) d s \\
(4.9) \pm: & \dddot{z}=z^{2} \pm \ddot{z}+A, \quad \text { multiply by } \dot{z} \text { and integrate } \\
\pm: & \dot{z} \ddot{z}-\frac{1}{3} z^{3} \mp \frac{1}{2} \dot{z}^{2}-A z=C+\int_{0}^{t} \ddot{z}^{2}(s) d s
\end{aligned}
$$

where $C$ is a constant. By Theorem 2.1 in [6], there is no bounded chaos in the systems.

\section{Chaotic candidates}

The following 21 systems which were not proved analytically are left as chaotic candidates.

$$
\begin{aligned}
& \left\{\begin{array}{l}
x^{\prime}=y^{2} \pm x+A z \\
y^{\prime}=x \\
z^{\prime}=y
\end{array}\right. \\
& \left\{\begin{array}{l}
x^{\prime}=y z \pm x+A y \\
y^{\prime}=z \\
z^{\prime}=x
\end{array}\right. \\
& \left\{\begin{array}{l}
x^{\prime}= \pm x+y+A z \\
y^{\prime}=x z \\
z^{\prime}=y
\end{array}\right. \\
& \left\{\begin{array}{l}
x^{\prime}=y^{2} \pm x \\
y^{\prime}=x+A z \\
z^{\prime}=y
\end{array}\right. \\
& \left\{\begin{array}{l}
x^{\prime}=y^{2}+A z \\
y^{\prime}=x \pm y \\
z^{\prime}=x
\end{array}\right. \\
& \left\{\begin{array}{l}
x^{\prime}=y^{2}+A z \\
y^{\prime}=x \pm y \\
z^{\prime}=y
\end{array}\right.
\end{aligned}
$$




$$
\begin{aligned}
& \left\{\begin{array}{l}
x^{\prime}=y^{2}+A z \\
y^{\prime}= \pm y+z \\
z^{\prime}=x
\end{array}\right. \\
& \left\{\begin{array}{l}
x^{\prime}=z^{2} \pm x \\
y^{\prime}=x+A z \\
z^{\prime}=y
\end{array}\right. \\
& \left\{\begin{array}{l}
x^{\prime}=y z \pm x \\
y^{\prime}=x+A z \\
z^{\prime}=y
\end{array}\right. \\
& \left\{\begin{array}{l}
x^{\prime}=y z+A y \\
y^{\prime}= \pm y+z \\
z^{\prime}=x
\end{array}\right. \\
& \left\{\begin{array}{l}
x^{\prime}=y z+A z \\
y^{\prime}=x \pm y \\
z^{\prime}=x
\end{array}\right. \\
& \left\{\begin{array}{l}
x^{\prime}=y z+A z \\
y^{\prime}=x \pm y \\
z^{\prime}=y
\end{array}\right. \\
& \left\{\begin{array}{l}
x^{\prime}=y z+A z \\
y^{\prime}= \pm y+z \\
z^{\prime}=x
\end{array}\right. \\
& \left\{\begin{array}{l}
x^{\prime}= \pm x+z \\
y^{\prime}=A y+z \\
z^{\prime}=x y
\end{array}\right. \\
& \left\{\begin{array}{l}
x^{\prime}= \pm x+y+A \\
y^{\prime}=x z \\
z^{\prime}=y
\end{array}\right. \\
& \left\{\begin{array}{l}
x^{\prime}= \pm x+z+A \\
y^{\prime}=x z \\
z^{\prime}=y
\end{array}\right. \\
& \left\{\begin{array}{l}
x^{\prime}=y z+A \\
y^{\prime}=x \pm y \\
z^{\prime}=x
\end{array}\right. \\
& \left\{\begin{array}{l}
x^{\prime}=y z+A \\
y^{\prime}= \pm y+z \\
z^{\prime}=x
\end{array}\right.
\end{aligned}
$$




$$
\begin{aligned}
& \left\{\begin{array}{l}
x^{\prime}=y z \pm x \\
y^{\prime}=z+A \\
z^{\prime}=x
\end{array}\right. \\
& \left\{\begin{array}{l}
x^{\prime}= \pm x+z \\
y^{\prime}=x+A \\
z^{\prime}=x y
\end{array}\right. \\
& \left\{\begin{array}{l}
x^{\prime}= \pm x+z \\
y^{\prime}=z+A \\
z^{\prime}=x y
\end{array}\right.
\end{aligned}
$$

where the "+" and "-" systems are labeled as $(n a)$ and $(n b)$ respectively. We can see that systems $(n b)$ are dissipative.

Theorem 5.1 (1) If $A \leqslant 0$ in system (3.1a) and $A \geqslant 0$ in system (3.1b);

(2) If $A \leqslant 0$ in system (3.6a) and $A \geqslant 0$ in system (3.6b);

(3) If $A \leqslant-1$ and $A=0$ in systems (3.11a) and $A \geqslant 1$ and $A=0$ in systems (3.11b);

(4) If $A \leqslant 0$ in system ( $3.19 a)$ and $A \geqslant 0$ in system (3.19b);

(5) If $A \geqslant 0$ in systems (3.13), (3.18), (3.22), (3.24), (3.31) and (3.35);

(6) If $A \leqslant 0$ in system ( $3.36 a)$ and $A \geqslant 0$ in system (3.36b);

(7) If $A \geqslant 1$ and $A=0$ in systems (3.44a) and $A \leqslant-1$ and $A=0$ in systems (3.44b), then these systems under the given conditions are not chaotic.

Proof: (1) From system (3.1), we have

$$
z^{\prime \prime \prime}=z^{\prime 2} \pm z^{\prime \prime}+A z
$$

Multiply by $z^{\prime \prime}$ and integrate to get

$$
z^{\prime \prime 2}-\frac{2}{3} z^{\prime 3}-2 A z z^{\prime}=C-2 \int_{0}^{t}\left(A z^{\prime 2} \mp z^{\prime \prime 2}\right) d s
$$

when $A \leqslant 0$ in system (3.1a) and $A \geqslant 0$ in system (3.1b), by Theorem 2.1 in [6], the system is not chaotic. (2) System (3.6) gives

$$
y^{\prime \prime \prime}=y y^{\prime} \pm y^{\prime \prime}+A y
$$

Multiply by $y$ and integrate to get

$$
y y^{\prime \prime}-\frac{1}{2} y^{\prime 2}-\frac{1}{3} y^{3} \mp y y^{\prime}=C+\int_{0}^{t}\left(A y^{2} \mp y^{\prime 2}\right) d s
$$

when $A \leqslant 0$ in system (3.6a) and $A \geqslant 0$ in system (3.1b), by Theorem 2.1 in [6], the system is not chaotic.

(3) Consider system $(3.11 a)$.

Since $(x-z)^{\prime}=x-z+(1+A) z$, let $u=x-z$. We have that $u^{\prime}=u+(1+A) z$, and $z^{\prime \prime}=x z$. Thus, $x=u+\frac{1}{1+A}\left(u^{\prime}-u\right)$. The scalar equation in $u$ is

Integrate to get

$$
u^{\prime \prime \prime}-u^{\prime \prime}=u u^{\prime}-u^{2}+\frac{1}{1+A}\left(u^{\prime}-u\right)^{2}
$$

$$
u^{\prime \prime}-u^{\prime}-0.5 u^{2}=C-\int_{0}^{t}\left(u^{2}(s)-\frac{1}{1+A}\left(u^{\prime}-u\right)^{2}\right) d s
$$


If $A<-1$, by Theorem 2.1 in [6], the system is not chaotic.

If $A=-1$, then $(x-z)^{\prime}=x-z$. We have that $x-z=C e^{t}$. When $C=0$, it is trivial. If $C>0$, then at least one of $x$ and $z$ is unbounded as $t \rightarrow \infty$. If $C<0$, the argument is similar to that for the case $C>0$. If $A=0$, integrate the first scalar equation in $u$ to get

$$
u^{\prime \prime}-u^{\prime}+\frac{1}{2} u^{2}=C+\int_{0}^{t} u^{\prime 2}(s) d s
$$

by Theorem 2.1 in [6], the system is not chaotic. Therefore system (3.11a) when $A \leqslant-1$ and $A=0$ is not chaotic.

Now consider system $(3.11 b)$. Since $(x-z)^{\prime}=-x+z+(A-1) z$, let $u=x-z$. We have that $u^{\prime}=-u+(A-1) z$, and $z^{\prime \prime}=x z$. Thus, $x=u+\frac{1}{A-1}\left(u^{\prime}+u\right)$. The scalar equation in $u$ is

$$
u^{\prime \prime \prime}+u^{\prime \prime}=u u^{\prime}+u^{2}+\frac{1}{A-1}\left(u^{\prime}+u\right)^{2}
$$

Integrate to get

$$
u^{\prime \prime}+u^{\prime}-0.5 u^{2}=C+\int_{0}^{t}\left(u^{2}(s)+\frac{1}{A-1}\left(u^{\prime}+u\right)^{2}\right) d s
$$

where $C$ is a constant. If $A>1$, by Theorem 2.1 in [6] system (3.11b) is not chaotic.

If $A=1,(x-z)^{\prime}=-x+z$. We have that $x-z=C e^{-t}$ and

$$
z^{\prime \prime}=x z=z^{2}+C e^{-t} z=\left(z+\frac{C}{2} e^{-t}\right)^{2}-\frac{C^{2}}{4} e^{-2 t}
$$

Integrate to get

$$
z^{\prime}=B+\frac{C^{2}}{8} e^{-2 t}+\int_{0}^{t}\left(z+\frac{C}{2} e^{-t}\right)^{2} d s
$$

where $C$ and $B$ are constants. Let $g(t)=\int_{0}^{t}\left(z+\frac{C}{2} e^{-t}\right)^{2} d s$. Then $g^{\prime}(t)>0$ for all $t>t_{1}>0$. Therefore $g(t)$ has a limit $L>-\infty$ and so $z^{\prime}$ has a limit. Hence system (3.11b) is not chaotic when $A=1$.

If $A=0$, integrate the scalar equation in $u$ to get

$$
u^{\prime \prime}+u^{\prime}+\frac{1}{2} u^{2}=C-\int_{0}^{t} u^{\prime 2}(s) d s
$$

where $C$ is a constant, by Theorem 2.1 in [6] the system is not chaotic. Therefore system $(3.11 b)$ when $A \geqslant 1$ and $A=0$ is not chaotic.

(4) Consider system (3.19).

Its two scalar Eq.s are $y^{\prime \prime \prime}=\left(y^{2}\right)^{\prime}+A y \pm y^{\prime \prime} \quad$ and $\quad z^{\prime \prime \prime}=z^{\prime 2}+A z \pm z^{\prime \prime}$. The second scalar Eq. is the same as that of (3.1). Multiply by $z^{\prime \prime}$. We get that

$$
z^{\prime \prime 2}-\frac{2}{3} z^{\prime 3}-2 A z z^{\prime}=C-2 \int_{0}^{t}\left(A z^{\prime 2} \mp z^{\prime \prime 2}\right) d s
$$

when $A \leqslant 0$ in system $(3.19 a)$ and $A \geqslant 0$ in system (3.19b), by Theorem 2.1 in [6], the systems are not chaotic. 
(5.1) Consider system (3.13).

The scalar Eq. of system (3.13) is $y^{\prime \prime \prime}=2 y y^{\prime}+A y^{\prime} \pm y^{\prime \prime} \mp A y$. Multiply by $y$ and integrate to get

$$
y y^{\prime \prime}-\frac{1}{2}\left(y^{\prime}\right)^{2}-\frac{2}{3} y^{3}-\frac{A}{2} y^{2} \mp y y^{\prime}=C \mp \int_{0}^{t}\left(\left(y^{\prime}(s)\right)^{2}+A y^{2}(s)\right) d s
$$

If $A \geqslant 0$, by Theorem 2.1 in [6], system (3.13) is not chaotic. Note that the scalar equation of system (3.13) in $z$ is $z^{\prime \prime \prime}=z^{\prime 2} \pm\left(z^{\prime \prime}-A z\right)+A z^{\prime}$.

Proposition 5.2 For system $(3.13 a)$, if $A<0$ and $x(0) \geqslant 0$, then $x(t)>0$ and $x^{\prime}(t) \geqslant 0$ for all $t \geqslant 0$.

Proof: If $x(0) \geqslant 0$, multiply the first Eq. of system $(3.13 a)$ by $e^{-t}$ to get $x=\left(x(0)+\int_{0}^{t} y^{2}(s) e^{-s} d s\right) e^{t}$. Obviously, $x^{\prime} \geqslant 0$, for $t \geqslant 0$. Hence (3.13a) with $A<0$ and $x(0) \geqslant 0$ is not chaotic.

(5.2) Consider system (3.18).

For $(3.18 a)$, let $u=y-z, u^{\prime}=y$. We have that

$$
u^{\prime \prime \prime}-u^{\prime \prime}=\left(u^{\prime}\right)^{2}+A u^{\prime}-A u
$$

Multiply by $u^{\prime \prime}$ and integrate to get

$$
\left(u^{\prime \prime}\right)^{2}-\frac{2}{3}\left(u^{\prime}\right)^{3}-A\left(u^{\prime}\right)^{2}+2 A u u^{\prime}=C+2 \int_{0}^{t}\left(\left(u^{\prime \prime}(s)\right)^{2}+A\left(u^{\prime}(s)\right)^{2}\right) d s
$$

For $(3.18 b)$, let $u=y-z, u^{\prime}=-y$. We have that

$$
u^{\prime \prime \prime}+u^{\prime \prime}=-\left(u^{\prime}\right)^{2}+A u^{\prime}+A u
$$

Multiply by $u^{\prime \prime}$ and integrate to get

$$
\left(u^{\prime \prime}\right)^{2}+\frac{2}{3}\left(u^{\prime}\right)^{3}-A\left(u^{\prime}\right)^{2}-2 A u u^{\prime}=C-2 \int_{0}^{t}\left(\left(u^{\prime \prime}(s)\right)^{2}+A\left(u^{\prime}(s)\right)^{2}\right) d s
$$

If $A \geqslant 0$, by Theorem 2.1 in [6], system (3.18) is not chaotic.

(5.3) Consider system (3.22).

The scalar Eq. of system (3.22) is $y^{\prime \prime \prime}= \pm y^{\prime \prime}+y^{2}+A y^{\prime} \mp y$ Multiply by $y^{\prime}$ and integrate

$$
y^{\prime} y^{\prime \prime} \mp \frac{1}{2}\left(y^{\prime}\right)^{2}-\frac{1}{3} \pm \frac{A}{2} y^{2}=C+\int_{0}^{t}\left(\left(y^{\prime \prime}(s)\right)^{2}+A\left(y^{\prime}(s)\right)^{2}\right) d s
$$

If $A \geqslant 0$, by Theorem 2.1 in [6], system (3.22) is not chaotic.

(5.4) Consider system (3.24).

Its scalar Eq. is $z^{\prime \prime \prime}= \pm z^{\prime \prime}+z^{2}+A z^{\prime} \mp z$ which is the same as that of (3.22). Hence systems (3.24) with $A \geqslant 0$ is not chaotic.

(5.5) Consider system (3.31).

The scalar Eq. of system (3.31) is $z^{\prime \prime \prime}= \pm z^{\prime \prime} \mp A z+z z^{\prime}+A z^{\prime}$. Multiply by $z$ and integrate

$$
z z^{\prime \prime}-\frac{1}{2}\left(z^{\prime}\right)^{2}-\frac{A}{2} z^{2}-\frac{1}{3} z^{3} \mp z z^{\prime}=C \mp \int_{0}^{t}\left(\left(z^{\prime}(s)\right)^{2}+A z^{2}\right) d s
$$

If $A \geqslant 0$, by Theorem 2.1 in [6], system (3.31) is not chaotic.

Let $x(0)=x_{0}, y(0)=y_{0}, z(0)=z_{0}$. 
Proposition 5.3 If $A<0$ and $x_{0}-\frac{1}{2} z_{0}^{2} \geqslant 0$, system (3.31a) is not chaotic.

Proof: By (3.31), $x^{\prime} \mp x=z z^{\prime}$ and $x=z^{\prime \prime}-A z$. Thus $\left(z^{\prime \prime}-A z\right)^{\prime} \mp\left(z^{\prime \prime}-A z\right)=\frac{1}{2}\left(z^{2}\right)^{\prime}$. Multiply both sides of the Eq. by $e^{\mp t}$ and integrate to get

$$
z^{\prime \prime}-A z-\frac{1}{2} z^{2}=C^{*} e^{ \pm t} \pm \frac{1}{2} e^{ \pm t} \int_{0}^{t} z^{2}(s) e^{\mp s} d s
$$

where $C^{*}=z^{\prime \prime}(0)-A z(0)-\frac{1}{2} z^{2}(0)=x_{0}-\frac{1}{2} z_{0}^{2}$.

If $A<0$ and $C^{*}=x_{0}-\frac{1}{2} z_{0}^{2} \geqslant 0$, for (3.31a) we have $z^{\prime \prime}-A z-\frac{1}{2} z^{2}=C^{*} e^{t}+\frac{1}{2} e^{t} \int_{0}^{t} z^{2}(s) e^{-s} d s$. Then $z^{\prime \prime}-A z-\frac{1}{2} z^{2} \rightarrow \infty$, as $t \rightarrow \infty$. This implies that $x=z^{\prime \prime}-A z \rightarrow \infty$ and either $z^{\prime \prime} \rightarrow \infty$ or $z \rightarrow \infty$, as $t \rightarrow \infty$. Hence $z \rightarrow \infty$.

(5.6) System (3.35) can be written as $z z^{\prime \prime \prime}-z^{\prime} z^{\prime \prime}= \pm z z^{\prime \prime} \mp A z^{2}+z^{2} z^{\prime}$. Integrate to get

$$
z z^{\prime \prime}-\left(z^{\prime}\right)^{2}-\frac{1}{3} z^{3} \mp z z^{\prime}=C \mp \int_{0}^{t}\left(\left(z^{\prime}(s)\right)^{2}+A z^{2}\right) d s
$$

If $A \geqslant 0$, by Theorem 2.1 in [6], system (3.35) is not chaotic.

(6) Consider system (3.36).

The scalar Eq. of (3.36) is $z^{\prime \prime \prime}=z z^{\prime}+A z \pm z^{\prime \prime}$. Multiply by $z$ and integrate

$$
z z^{\prime \prime}-\frac{1}{2} z^{\prime 2}-\frac{1}{3} z^{3} \mp z z^{\prime}=C+\int_{0}^{t}\left(A z^{2} \mp z^{\prime 2}\right) d s
$$

where $C$ is a constant and $t \geqslant 0$. When $A \leqslant 0$ in (3.36a) and $A \geqslant 0$ in (3.36b), by Theorem 2.1 in [6], system (3.36) is not chaotic.

(7) Consider system (3.44).

For system (3.44a), if $A=1$, then $x^{\prime}-y^{\prime}=x-y, x-y=C e^{t}$. So at least one of $x$ and $y$ is unbounded. Hence the system is not chaotic.

If $A \neq 1$, then $(x-y)^{\prime}=x-A y=(x-y)+(1-A) y$. Let $u=x-y$. Then $u^{\prime}=u+(1-A) y$. Since $x=u+y$, we have $y^{\prime \prime}=A y^{\prime}+x y$. This implies

$$
u^{\prime \prime \prime}-(1+A) u^{\prime \prime}=-A u^{\prime}+u u^{\prime}-u^{2}-\frac{1}{1-A}\left(u^{\prime}-u\right)^{2}
$$

Integrate to get

$$
u^{\prime \prime}-(1+A) u^{\prime}+A u-\frac{1}{2} u^{2}=C-\int_{0}^{t}\left(u^{2}-\frac{1}{1-A}\left(u^{\prime}-u\right)^{2}\right) d s
$$

and case $A=0$, we have

$$
u^{\prime \prime}-u^{\prime}-\frac{3}{2} u^{2}=C-\int_{0}^{t}\left(2 u^{2}+\left(u^{\prime}\right)^{2}\right) d s
$$

If $A>1$ and $A=0$, by Theorem 2.1 in [6], system (3.44a) is not chaotic.

Consider system (3.44b).

If $A=-1, x^{\prime}-y^{\prime}=-(x-y)$, then $x-y=C e^{-t}$. Thus, $y^{\prime \prime}+y^{\prime}=y^{2}+C e^{-t} y=\left(y+\frac{C e^{-t}}{2}\right)^{2}-\frac{C^{2} e^{-2 t}}{4}$, where $C=x_{0}-y_{0}$. Let $\hat{y}=y+\frac{C e^{-t}}{2}$. Then $\hat{y}^{\prime \prime}+\hat{y}^{\prime}=y^{\prime \prime}+y^{\prime}=\hat{y}^{2}-\frac{C^{2} e^{-2 t}}{4}$. 
Integrate to get

$$
\hat{y}^{\prime}+\hat{y}=C_{1}+\frac{C^{2}}{8}\left(e^{-2 t}-1\right)+\int_{0}^{t} \hat{y}^{2}(s) d s
$$

where $C_{1}=\hat{y}^{\prime}(0)+\hat{y}(0)=z_{0}$. Let $g(t)=\int_{0}^{t} \hat{y}^{2}(s) d s>0$. Then $g^{\prime} \geqslant 0$, for all $t \geqslant 0$ and so $g$ has a limit $L>0$. If $L=\infty$, the solution is unbounded. If $L<\infty, \hat{y}^{\prime}+\hat{y} \rightarrow C_{1}-\frac{C^{2}}{8}+L=K$, as $t \rightarrow \infty$. We define sets of two 2-dimensional surfaces as follows:

$$
\begin{aligned}
& S_{1}=\left\{(x, y, z) \in \mathbb{R}^{3}: x-y=0\right\} \\
& S_{2}=\left\{(x, y, z) \in \mathbb{R}^{3}: z=K\right\}
\end{aligned}
$$

$\hat{y}^{\prime}+\hat{y} \rightarrow y^{\prime}+y=z \rightarrow K$, imply that $(x, y, z) \rightarrow S_{2}$, and $(x, y, z) \rightarrow S_{1}$. So $(x, y, z) \rightarrow S_{1} \cap S_{2}$, as $t \rightarrow \infty$. Hence system (3.44b) with $A=-1$ is not chaotic.

If $A \neq-1,(x-y)^{\prime}=-x-A y=-(x-y)-(1+A) y$. Let $u=x-y$. Then $u^{\prime}=-u-(1+A) y$. Since $x=u+y, y^{\prime \prime}=A y^{\prime}+x y$. We have

$$
u^{\prime \prime \prime}+(1-A) u^{\prime \prime}=A u^{\prime}+u u^{\prime}+u^{2}-\frac{1}{1+A}\left(u^{\prime}+u\right)^{2}
$$

Integrate to get

$$
u^{\prime \prime}+(1-A) u^{\prime}-A u-\frac{1}{2} u^{2}=C+\int_{0}^{t}\left(u^{2}-\frac{1}{1+A}\left(u^{\prime}-u\right)^{2}\right) d s
$$

When $A=0$, we have

$$
u^{\prime \prime}+u^{\prime}+\frac{1}{2} u^{2}=C-\int_{0}^{t}\left(u^{\prime}\right)^{2} d s
$$

If $A<-1$ and $A=0$, by Theorem 2.1 in [6], system (3.44b) is not chaotic.

Proposition 5.4 (1) Systems (3.34), (3.37) and (4.6) are equivalent.

(2) System (3.35) is equivalent to systems (4.5) and (4.21).

Proof. (1) System (3.34), after the transformation $\bar{z}=z+A, \bar{y}=y, \bar{x}=x$, has the following form:

$$
\bar{x}^{\prime}=\bar{y} \bar{z}, \quad \bar{y}^{\prime}= \pm \bar{y}+\bar{z}-A, \quad \bar{z}^{\prime}=\bar{x}
$$

System (3.37) with $\bar{z}=z, \bar{y}=y+A, \bar{x}=x$ becomes (5.1) and system (4.6) with $x=\bar{y}, y=\bar{x}$ and $z=\bar{z}$ becomes (5.1).

(2) System (4.5) after the transformation $\bar{z}=z, \bar{y}=y+A, \bar{x}=x$, becomes $\bar{x}^{\prime}= \pm \bar{x}+\bar{y}, \bar{y}^{\prime}=\bar{x} \bar{z}$, $\bar{z}^{\prime}=\bar{y}-A$ Let $Y=\bar{z}, Z=\bar{y}$ and $X=\bar{x}$ to get $X^{\prime}= \pm X+Z, Y^{\prime}=Z-A, Z^{\prime}=X Y$. We obtain (4.21).

Starting from system (4.5a), we let $X=A+x, Y=y$ and $Z=z$ to get $X^{\prime}=X+Y, Y^{\prime}=X Z-A Z$, $Z^{\prime}=Y$. Exchange $X$ and $Y$, we have $X^{\prime}=Y Z-A Z, Y^{\prime}=X+Y, Z^{\prime}=X$. We get (3.35a).

Starting from system (4.5b), let $X=A-x, Y=y$ and $Z=z$. We have $X^{\prime}=-X-Y, Y^{\prime}=A Z-X Z$, $Z^{\prime}=Y$. Let $X=-v, Y=u$ and $Z=w$. Then the system becomes

$$
u^{\prime}=v w+A w, \quad v^{\prime}=u-v, \quad w^{\prime}=u
$$

Change the names $u, v$ and $w$ back to $x, y$ and $z$ we get (3.35b).

Systems (3.35) and (4.6) will be studied later. 
Theorem 5.5 (1) If $A \leqslant 0$ in system (4.5a) and $A \geqslant 0$ in system (4.5b);

(2) If $A \geqslant 0$ in system (4.10);

(3) If $A \leqslant 0$ in system (4.12a) and $A \geqslant 0$ in system (4.12b);

(4) If $A \geqslant 0$ in system (4.17a) and $A \leqslant 0$ in system (4.17b);

(5) If $A \geqslant 0$ in system (4.20a) and $A \leqslant 0$ in system (4.20b);

(6) If $A \geqslant 0$ in system (4.21a) and $A \leqslant 0$ in system (4.21b);

then these systems under the given conditions are not chaotic.

Proof: (1) Consider system (4.5).

Since $y^{\prime \prime}=x^{\prime} z+x z^{\prime}=( \pm x+y+A) z+x y$, we have

$$
y^{\prime \prime}= \pm y^{\prime}+y z+A z+x y \quad \text { or } \quad z^{\prime \prime \prime}= \pm z^{\prime \prime}+z z^{\prime}+A z+z^{\prime} x
$$

Multiply the Eq. in $z$ by $z$ to get

$$
z z^{\prime \prime \prime}= \pm z z^{\prime \prime}+z^{2} z^{\prime}+A z^{2}+z^{\prime} z^{\prime \prime}
$$

Integrate to get

$$
z z^{\prime \prime}-z^{\prime 2}-\frac{1}{3} z^{3} \mp z z^{\prime}=C+\int_{0}^{t}\left(A z^{2} \mp z^{\prime 2}\right) d s
$$

where $C$ is a constant and $t \geqslant 0$. When $A \leqslant 0$ in (4.5a) and $A \geqslant 0$ in (4.5b), by Theorem 2.1 in [6], system (4.5) is not chaotic.

(2) Consider system (4.10).

$$
\begin{gathered}
y^{\prime \prime}=y z+A \pm y^{\prime} \cdot y^{\prime \prime \prime}=y^{\prime} z+y z^{\prime} \pm y^{\prime \prime}=y^{\prime} z+y x \pm y^{\prime \prime} \\
y^{\prime \prime \prime}=y^{\prime} z+y\left(y^{\prime} \mp y\right) \pm y^{\prime \prime}, \quad \text { or } \quad y y^{\prime \prime \prime}=y^{\prime}\left(x^{\prime}-A\right)+y^{2}\left(y^{\prime} \mp y\right) \pm y y^{\prime \prime} \\
y y^{\prime \prime \prime}=y^{\prime}\left(y^{\prime \prime} \mp y^{\prime}\right)-A y^{\prime}+y^{2} y^{\prime} \mp y^{3} \pm y y^{\prime \prime}
\end{gathered}
$$

From $(4.10 a)$, we have

$$
\begin{gathered}
-x^{\prime}=-y z-A, \quad z y^{\prime}=x z+y z, \quad-z z^{\prime}=-x z \\
y z^{\prime}=x y, \quad-y y^{\prime}=-x y-y^{2}
\end{gathered}
$$

Then

$$
\begin{gathered}
\left(-x-\frac{1}{2} z^{2}-\frac{1}{2} y^{2}+y z\right)^{\prime}=-A-y^{2} \\
-x-\frac{1}{2} z^{2}-\frac{1}{2} y^{2}+y z=C-\int_{0}^{t}\left(A+y^{2}\right) d s
\end{gathered}
$$

where $C$ is a constant and $t \geqslant 0$. When $A \geqslant 0$, by Theorem 2.1 in [6], system (4.10a) is not chaotic.

From (4.10b), we have

$$
\begin{gathered}
x^{\prime}=y z+A, \quad z y^{\prime}=x z+y z, \quad-z z^{\prime}=-x z \\
y z^{\prime}=x y, \quad-y y^{\prime}=-x y+y^{2}
\end{gathered}
$$

Then

$$
\begin{gathered}
\left(x-\frac{1}{2} z^{2}-\frac{1}{2} y^{2}+y z\right)^{\prime}=A+y^{2} \\
x-\frac{1}{2} z^{2}-\frac{1}{2} y^{2}+y z=C+\int_{0}^{t}\left(A+y^{2}\right) d s
\end{gathered}
$$


where $C$ is a constant and $t \geqslant 0$. When $A \geqslant 0$, by Theorem 2.1 in [6], system (4.10b) is not chaotic.

(3) Consider system (4.12).

The scalar equation of (4.12) is: $y^{\prime \prime \prime}= \pm y^{\prime \prime}+y y^{\prime} \mp y^{2}+A$. Integrate to get

$$
y^{\prime \prime} \mp y^{\prime}-0.5 y^{2}=C+\int_{0}^{t}\left(A \mp y(s)^{2}\right) d s
$$

where $C$ is a constant and $t \geqslant 0$. When $A \leqslant 0$ in (4.12a) and $A \geqslant 0$ in (4.12b), by Theorem 2.1 in [6], system (4.12) is not chaotic.

(4) Consider system (4.17).

The scalar Eq. of (4.17) is: $y^{\prime \prime \prime}=y y^{\prime}-A y \pm y^{\prime \prime}$, multiply by $y$ to get

$$
y y^{\prime \prime}-\frac{1}{2} y^{\prime 2} \mp y y^{\prime}-\frac{1}{3} y^{3}=C-\int_{0}^{t}\left(A y(s)^{2} \pm y^{\prime}(s)^{2}\right) d s
$$

where $C$ is a constant and $t \geqslant 0$. When $A \leqslant 0$ in (4.17a) and $A \geqslant 0$ in (4.17b), by Theorem 2.1 in [6], system (4.17) is not chaotic.

(5) Consider system (4.20).

The scalar equation in $y$ is

$$
y^{\prime \prime \prime}= \pm y^{\prime \prime}+y y^{\prime}-A y
$$

Integrate to get

$$
y y^{\prime \prime}-\frac{1}{2} y^{\prime 2}=y y^{\prime}+\frac{1}{3} y^{3}-\int\left(y^{\prime 2} \pm A y^{2}\right) d s+C
$$

by Theorem 2.1 in [6], system (4.20a) when $A \geqslant 0$ and system (4.20b) when $A \leqslant 0$ are not chaotic.

(6) Consider system (4.21).

Since $y^{\prime \prime}=x y, y^{\prime \prime \prime}=x^{\prime} y+y x^{\prime}=( \pm x+z) y+y^{\prime} x=\left( \pm x+y^{\prime}-A\right) y+y^{\prime} x$. The scalar Eq. of (4.21) is

$$
y y^{\prime \prime \prime}= \pm y y^{\prime \prime}+y^{2} y^{\prime}-A y^{2}+y^{\prime} y^{\prime \prime}
$$

Integrate to get

$$
y y^{\prime \prime}-y^{\prime 2} \mp y y^{\prime}-\frac{1}{3} y^{3}=C+\int_{0}^{t}\left(-A y(s)^{2} \mp y^{\prime}(s)^{2}\right) d s
$$

where $C$ is a constant and $t \geqslant 0$. When $A \geqslant 0$ in (4.21a) and $A \leqslant 0$ in (4.21b), by Theorem 2.1 in [6], system (4.21) is not chaotic.

Now we consider system (4.6). Since $z^{\prime \prime}=x z, z=x^{\prime} \mp x-A$, we have

$$
x^{\prime \prime \prime} \mp x^{\prime \prime}=x x^{\prime} \mp x^{2}-A x
$$

Our method doesn't work for this scalar equation. We will give numerical results on this system in section 7 .

Proposition 5.6 System (4.6a) has an invariant region for $A \leqslant 0: \Omega_{1}=\{(x, y, z) \mid x+z+A \geqslant 0, y>$ $0, z>0\}$. 
The proof is obvious.

Among the 21 systems in this section, 9 of them exhibit chaos. For the rest 12 systems after a large amount of numerical simulations we didn't find chaotic behavior in them. In the next two sections, we will give some of our numerical results on the systems including chaotic and nonchaotic behavior.

\section{Simplest dissipative chaotic systems}

Nine out of the 21 chaotic candidates in section 5 exhibit chaos. They are systems (3.1), (3.6), (3.19), (3.35), (3.36), (4.5), (4.17), (4.20), and (4.21). By Proposition 5.4 systems (3.35), (4.5) and (4.21) are linearly equivalent. After the following scalar transformations the " $b$ " systems are transformed to the " $a$ " systems for the following seven systems:

$$
\begin{array}{ll}
x=-X, y=Y, z=-Z, t=-\tau & (3.1 b) \rightarrow(3.1 a) \text { with } A \text { replaced by }-A \\
x=X, y=Y, z=-Z, t=-\tau & (3.6 b) \rightarrow(3.6 a) \text { with } A \text { replaced by }-A \\
x=-X, y=Y, z=-Z, t=-\tau & (3.19 b) \rightarrow(3.19 a) \text { with } A \text { replaced by }-A \\
x=X, y=-Y, z=Z, t=-\tau & (3.36 b) \rightarrow(3.36 a) \\
x=X, y=Y, z=-Z, t=-\tau & (4.17 b) \rightarrow(4.17 a) \text { with } A \text { replaced by }-A \\
x=-X, y=Y, z=Z, t=-\tau & (4.20 b) \rightarrow(4.20 a) \text { with } A \text { replaced by }-A \\
x=-X, y=Y, z=Z, t=-\tau & (3.35 b) \rightarrow(3.35 a)
\end{array}
$$

Therefore if a " $b$ " system has chaos when $t \rightarrow \infty$, then the corresponding " $a$ " system has chaos when $t \rightarrow-\infty$ and vice versa.

The scalar equations of the seven " $b$ " systems are:

(3.1b), (3.19b) $y^{\prime \prime \prime}+y^{\prime \prime}-2 y y^{\prime}-A y=0$

(3.6b) $\quad y^{\prime \prime \prime}+y^{\prime \prime}-y y^{\prime}-A y=0$

(3.36b) $\quad z^{\prime \prime \prime}+z^{\prime \prime}-z z^{\prime}-A z=0$

(4.17b), (4.20b) $y^{\prime \prime \prime}+y^{\prime \prime}-y y^{\prime}+A y=0$

(3.35b) $\quad z z^{\prime \prime \prime}-z^{\prime} z^{\prime \prime}=-z z^{\prime \prime}+A z^{2}+z^{2} z^{\prime}$

Each of the above scalar equations except that of (3.35b) is linearly equivalent to system (14) $Y^{\prime \prime \prime}+$ $a Y^{\prime \prime} \pm Y Y^{\prime}+Y=0$ in [13] (Sprott, 2000), when $a>0$ the system is dissipative. In 3D Sprott's system reads

$$
Y^{\prime}=Z, \quad Z^{\prime}=X, \quad X^{\prime}=\mp Y Z-a X-Y, \quad '=\frac{d}{d \tau}
$$

In $3 \mathrm{D}$ the scalar equations of $(3.6 \mathrm{~b})$ reads

$$
y^{\prime}=z, \quad z^{\prime}=x, \quad x^{\prime}=y z-x+A y, \quad '=\frac{d}{d t}
$$

After the transformations

$$
X=\mp a^{4} x, \quad Y=\mp a^{2} y, \quad Z=\mp a^{3} z, \quad \tau=\frac{1}{a} t
$$

Sprott's 3D system is transformed to the above 3D system and therefore Sprott's scalar equation becomes the scalar equation of $(3.6 b) y^{\prime \prime \prime}-y y^{\prime}-A y+y^{\prime \prime}=0$ with $A=-\frac{1}{a^{3}}$. Similarly after the transformation

$$
X=\mp 2 a^{4} x, \quad Y=\mp 2 a^{2} y, \quad Z=\mp 2 a^{3} z, \quad \tau=\frac{1}{a} t
$$


Sprott's scalar equation becomes the scalar equation of (3.1b) $y^{\prime \prime \prime}-2 y y^{\prime}-A y+y^{\prime \prime}=0$ with $A=-\frac{1}{a^{3}}$. For the scalar equation of (3.36b) we simply have to switch the names of $y$ and $z$. For the scalar equation of (4.17b), and (4.20b) we let $A=\frac{1}{a^{3}}$. To study the chaotic behavior of these systems, it is sufficient to study system $(3.1 b)$ where chaos was discovered by Sprott [10] and system $(3.35 b)$ where chaos was discovered by Melasoma [8].

It is well known that sensitivity on initial conditions of bounded solutions implies chaos and it is characterized by the Lyapunov spectrum or the largest Lyapunov exponent (usually base $e$ ):

$$
|\Delta Z(t)| \approx e^{\lambda t}\left|\Delta Z_{0}\right|
$$

where $\Delta Z_{0}$ is the initial separation.

For readers' convenience we give the definitions of Lyapunov exponents. From [1] the Lyapunov exponents of the orbit starting from a point $v_{0}$ of a smooth map $f$ is defined as:

Let $f: \mathbb{R}^{m} \rightarrow \mathbb{R}^{m}$ be a smooth map, let $J_{n}=D f^{n}\left(v_{0}\right)$ be the first derivative of the $n t h$ iterate of $f$, where $v_{0} \in \mathbb{R}^{m}$ and for $k=1,2, \ldots, m$, let $r_{k}^{n}$ be the $k$ th longest axis of the ellipsoid $J_{n} U$, where $U$ is the unit sphere centered at $v_{0}$. Then $r_{k}^{n}$ measures the contraction or expansion near the orbit of $v_{0}$ during the first $n$ iterations. The $k t h$ Lyapunov number of $v_{0}$ is defined by

$$
L_{k}=\lim _{n \rightarrow \infty}\left(r_{k}^{n}\right)^{1 / n}
$$

if this limit exists. The kth Lyapunov exponent of $v_{0}$ is $h_{k}=\ln L_{k}$.

Usually for a continuous dynamical systems, the map is chosen as a Poincaré section, say $\{(x, y, z) \in$ $\left.\mathbb{R}^{3} \mid z=0, y>0\right\}$.

Another character of chaos in dissipative systems is that they can have strange attractors, which can possess a non-integer dimension. From [1] and [3] the Kaplan-Yorke or Lyapunov dimension is defined as:

Let $f: \mathbb{R}^{m} \rightarrow \mathbb{R}^{m}$ be a smooth map. Consider an orbit with Lyapunov exponents $h_{1} \geqslant h_{2} \geqslant \ldots \geqslant h_{m}$, and let $p$ denote the largest integer such that

$$
\sum_{i=1}^{p} h_{i} \geqslant 0
$$

Define the Kaplan-Yorke or Lyapunov dimension $D_{L}$ of the orbit by

$$
D_{L}= \begin{cases}0 & \text { if no such p exists } \\ p+\frac{1}{\left|h_{p+1}\right|} \Sigma_{i=1}^{p} h_{i} & \text { if } p<m \\ m & \text { if } p=m\end{cases}
$$

Chaotic solutions in the first six systems (3.1b) to (4.20b) are shown in Figures 1 to 4 and Figures 8 and 9. Another scalar equation for (3.1b) is $z^{\prime \prime \prime}-z^{\prime 2}-A z+z^{\prime \prime}=0$. After the transformation

$$
X=a x, \quad Y=a^{2} y, \quad Z=a^{3} z, \quad \tau=a t
$$

Sprott's ([11], 1997) equation $Z^{\prime \prime \prime}+a Z^{\prime \prime}-Z^{\prime 2}+Z=0$ becomes $(3.1 b)$ 's $z^{\prime \prime \prime}-z^{\prime 2}-A z+z^{\prime \prime}=0$ with $A=-\frac{1}{a^{3}}$. From [11] for $2.0168<a<2.0577$ the systems exhibit chaos. When $a=2.017$ the Lyapunov exponents of an orbit is $(0.0550,0,-2.0720)$ and the corresponding Kaplan-Yorke dimension is $D_{K Y}=2.0265$. According to the rescaling when $-0.121902 \ldots<A<-0.114767 \ldots$ system $(3.1 b)$ exhibit chaos. When $A=0.121866$ system $(3.1 b)$ has a positive Lyapunov exponent and non-integer dimension. Due to the equivalence of the six systems, there are chaotic solutions in all the six systems. 


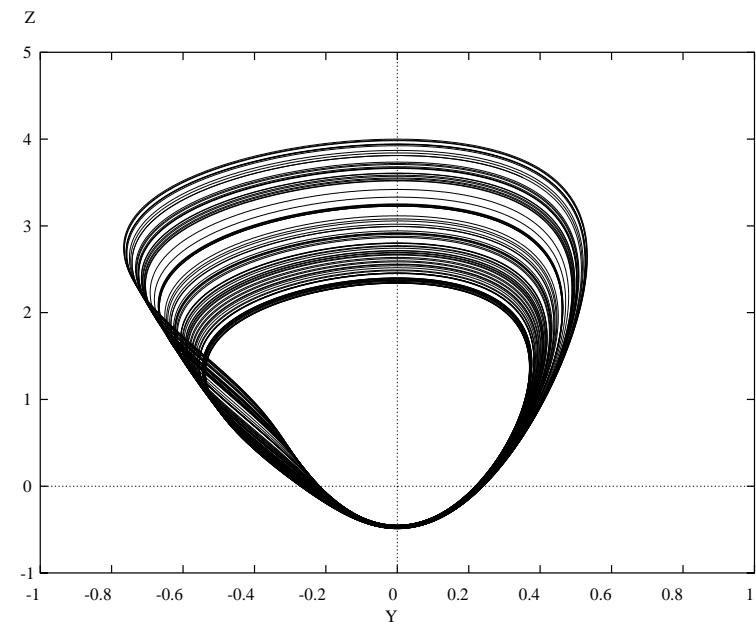

Figure 1: A strange attractor of system $(3.1 b)$ $A=-0.12177, x_{0}=-0.00228, y_{0}=0.034324$, $z_{0}=0.134228$

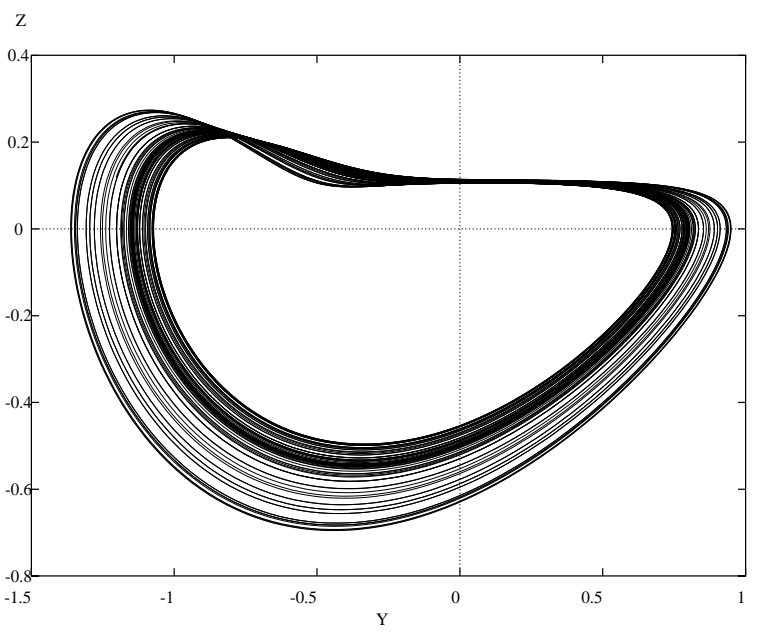

Figure 2: A strange attractor of system (3.6b) $A=-0.117, x_{0}=0.23421, y_{0}=-1.20288, z_{0}=0.118738$

In two of the first six systems we show pictures of stable period 1, 2, 4, 8, and 12 orbits in Figures 5 to 7 and 10 to 12 . It seems that the route to chaos while parameters change in the systems is through the increase of periods of periodic orbits. The existence of periodic solutions in continuous systems may have an order similar to the Sharkovskii Theorem.

The characteristic polynomial corresponds to the linearization of $(3.1 \mathrm{~b})$ at the equilibrium $(0,0,0)$ of the system is $\lambda^{3}+\lambda^{2}-A=0$, where $A<0$. The characteristic polynomials of the first four systems are the same. The characteristic polynomial for systems (4.17b) and (4.20b) is the same as that of $(3.1 \mathrm{~b})$ except the sign of the parameter $A$. Let $\lambda=\xi-\frac{1}{3}$. Then the polynomial becomes $\xi^{3}-\frac{1}{3} \xi+\frac{2}{27}-A$. Let $\omega=-\frac{1}{2}+\frac{\sqrt{3}}{2} i$ and so $\omega^{2}=-\frac{1}{2}-\frac{\sqrt{3}}{2} i$. Then the three roots of the polynomial are

$$
\begin{gathered}
\lambda_{1}=-\frac{1}{3}+\left\{\frac{A}{2}-\frac{1}{27}+\sqrt{A(A / 4-1 / 27)}\right\}^{1 / 3}+\left\{\frac{A}{2}-\frac{1}{27}-\sqrt{A(A / 4-1 / 27)}\right\}^{1 / 3} \\
\lambda_{2}=-\frac{1}{3}+\omega\left\{\frac{A}{2}-\frac{1}{27}+\sqrt{A(A / 4-1 / 27)}\right\}^{1 / 3}+\omega^{2}\left\{\frac{A}{2}-\frac{1}{27}-\sqrt{A(A / 4-1 / 27)}\right\}^{1 / 3} \\
\lambda_{3}=-\frac{1}{3}+\omega^{2}\left\{\frac{A}{2}-\frac{1}{27}+\sqrt{A(A / 4-1 / 27)}\right\}^{1 / 3}+\omega\left\{\frac{A}{2}-\frac{1}{27}-\sqrt{A(A / 4-1 / 27)}\right\}^{1 / 3}
\end{gathered}
$$

So the discriminant $\Delta=A(A / 4-1 / 27)$ is positive when $A<0$ and it has a negative real root and two complex roots for all $A<0$. This implies that solutions oscillate near the equilibrium.

All the first four systems (3.1a) to (3.36a) have a unique equilibrium $(0,0,0)$ with the same characteristic polynomial $\lambda^{3}-\lambda^{2}-A$. The characteristic polynomial for systems (4.17a) and (4.20a) is the same as that of (3.1a) except the sign of the parameter $A$. Let $\lambda=\xi+\frac{1}{3}$. Then the polynomial becomes $\xi^{3}-\frac{1}{3} \xi-A-\frac{2}{27}$ and the discriminant is $\Delta=A(A / 4+1 / 27)$. When $A>0$ it has a positive real root and two complex conjugate roots. The solution of system (3.1a) with $A>0$ shown in Figure 13 is a relatively complicated one that we can find. Most solutions become monotone quickly as $t$ increases.

The behavior of systems (3.6a), (3.19a), (3.36a) all three with $A>0$, and systems $(4.17 a)$ and $(4.20 a)$ both with $A<0$ are similar to those of system (3.1a).

Now we look at system (3.35) with $A<0$. The characteristic polynomials of the equilibrium $(0,0,0)$ are $( \pm 1-\lambda)\left(\lambda^{2}-A\right)$ for the " $a$ " and " $b$ " systems respectively. It was discovered by Malasoma ([8], 2002) 




Figure 3: A strange attractor of system (3.19b) $A=-0.117, x(0)=-0.0832, y(0)=-0.133358$, $z(0)=-0.28375$

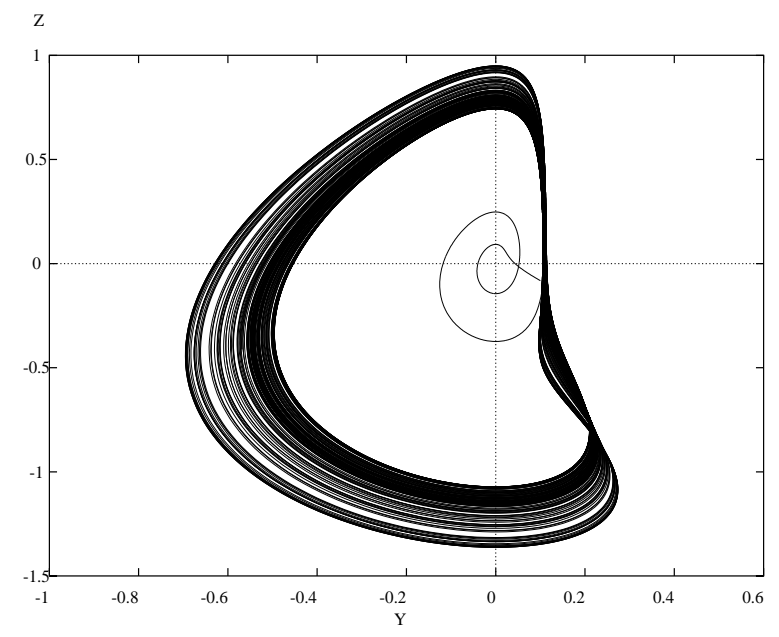

Figure 4: A strange attractor of system (3.36b) $A=-0.117, x(0)=0.0192, y(0)=0.1, z(0)=-0.082$.

that it exhibits chaos in a very narrow interval of the parameter and initial conditions. After switching $X$ and $Z$ in Malasoma's system M(11) (see the last section) and then the scalar transformation

$$
X=\alpha^{3} x, \quad Y=\alpha^{2} y, \quad Z=\alpha z, \quad \tau=\frac{1}{\alpha} t
$$

Malasoma's system $\mathrm{M}(11)$ is transformed to system (3.35) with $A=-\frac{1}{\alpha^{2}}$. Malasoma found chaos when $10.2849<\alpha<10.3716$, i.e. $-0.00945365726<A<-0.0092962647$. When $\alpha=10.285$, i.e. $A=0.0094534734$, the system has s solution with a positive Lyapunov exponent greater than 0.01 and Kaplan-Yorke dimension $D_{K Y} \approx 2.01$. Figure 14 shows a chaotic attractor of system (3.35). 


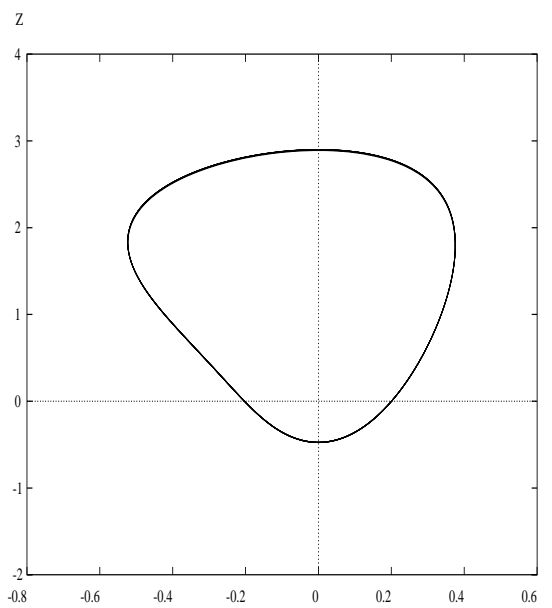

$(a)^{Y}$

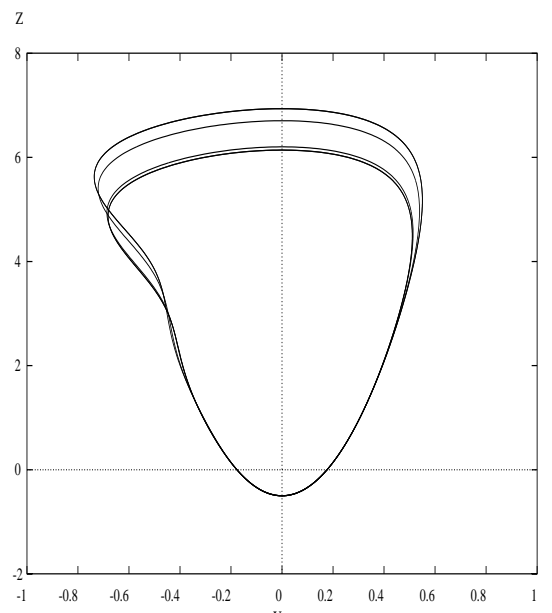

$(c)^{Y}$

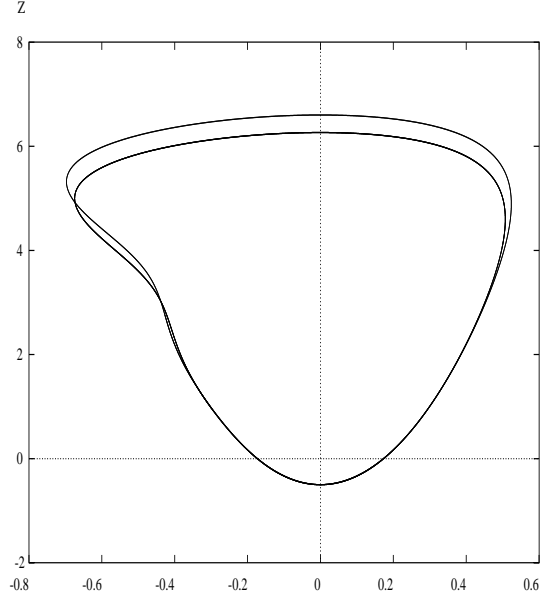

(b)

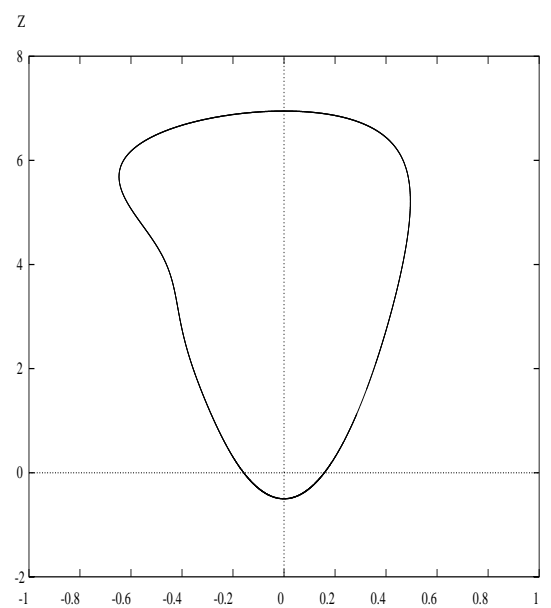

$(d)^{y}$

Figure 5: System (3.1b)'s periodic orbits

(a) Small period one $A=-0.091, x_{0}=0.0424, y_{0}=0.145, z_{0}=-0.228$

(b) Period two $A=-0.06, x_{0}=0.014587, y_{0}=0.4984839, z_{0}=4.054846$

(c) Period four $A=-0.0625, x_{0}=-0.3251857, y_{0}=-0.3382, z_{0}=6.02942896$

(d) Big period one $A=-0.05, x_{0}=0.0244992, y_{0}=0.3598, z_{0}=2.103894$ 


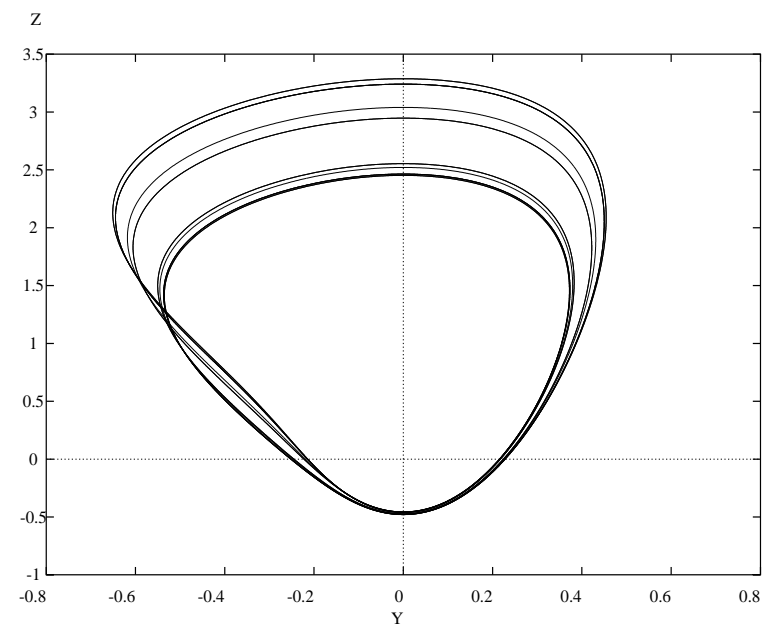

Figure 6: System (3.1b) period eight solution. $A=-0.1146, x_{0}=0.0484239, y_{0}=0.2239523$, $z_{0}=0.0378952$



Figure 8: A strange attractor of system (4.17b) $A=0.117, x(0)=1.00213, y(0)=1.34918$, $z(0)=-0.744$

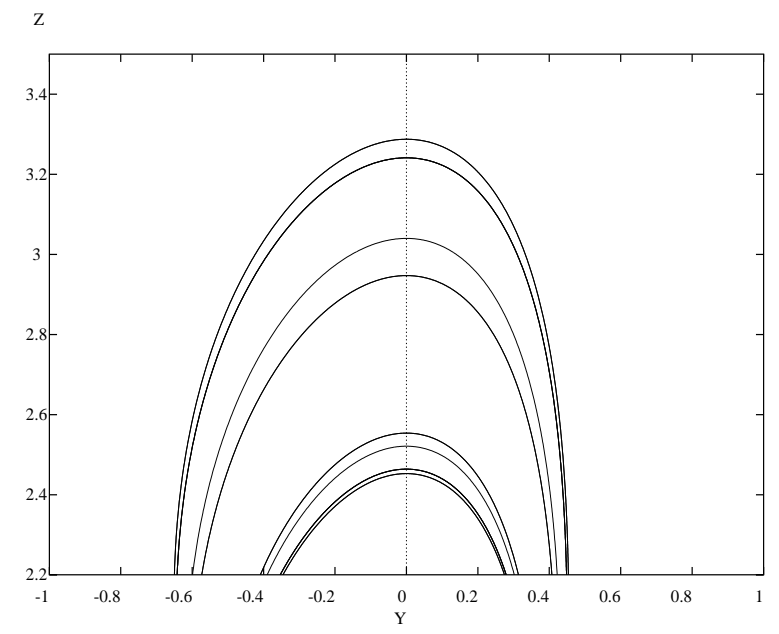

Figure 7: System (3.1b) period eight solution. $A=-0.1146, x_{0}=0.0484239, y_{0}=0.2239523$, $z_{0}=0.0378952$

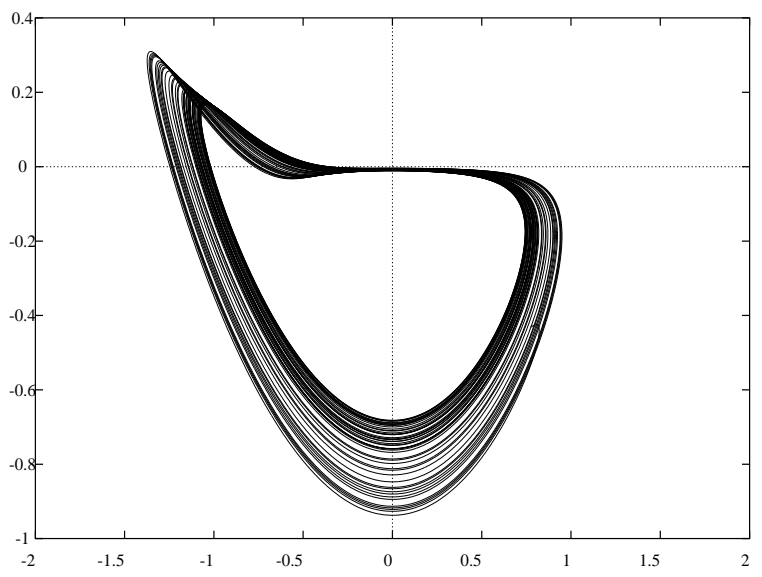

Figure 9: A strange attractor of system (4.20b) $A=0.117, x(0)=0.0902, y(0)=0.773467$, $z(0)=-0.432$ 




(a)

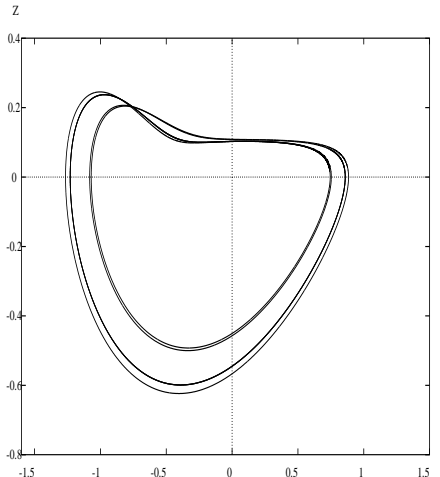

(c)

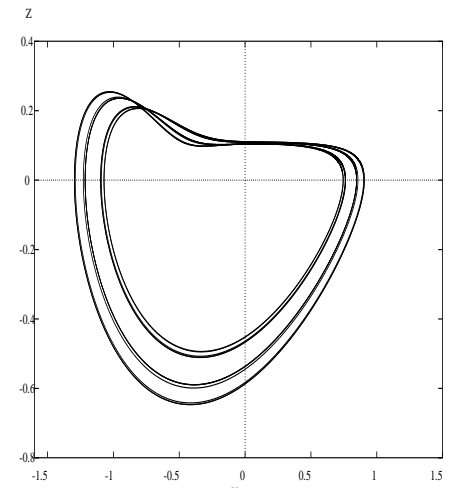

$(\mathrm{e})^{\gamma}$

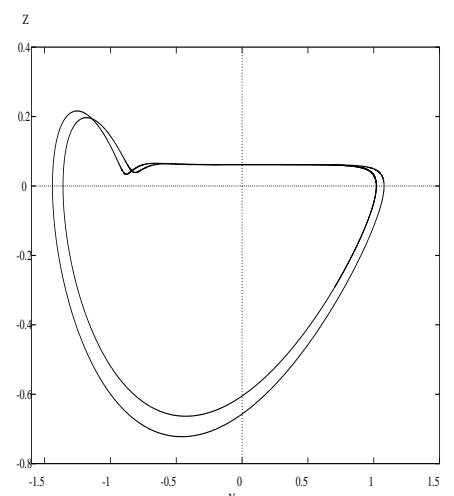

(b)

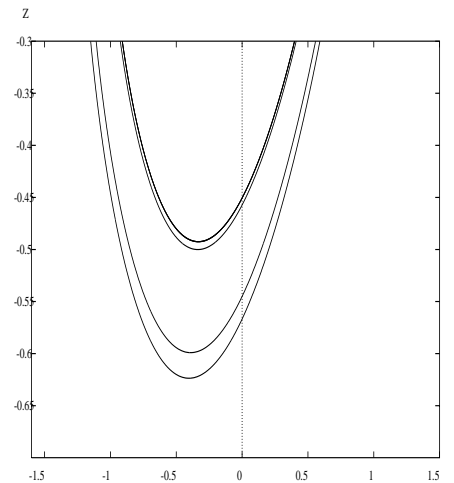

(d)

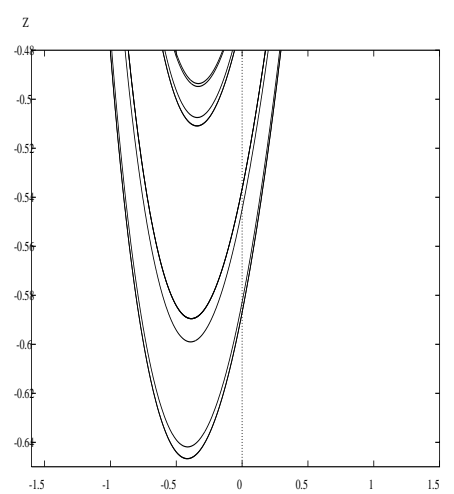

$(f)^{r}$

Figure 10: System (3.6b)'s periodic orbits

(a) Period one $A=-0.0517, x_{0}=-0.019754, y_{0}=-1.1067, z_{0}=0.1654435$

(b) Period two $A=-0.061708, x_{0}=0.0006, y_{0}=0.69191, z_{0}=0.060376$

(c), (d) Period four $A=-0.11295, x_{0}=-0.0158, y_{0}=0.744694, z_{0}=0.07963$

(e), (f) Period eight $A=-0.1143783, x_{0}=-0.01984643, y_{0}=-0.35572886$, $z_{0}=0.1326593$ 


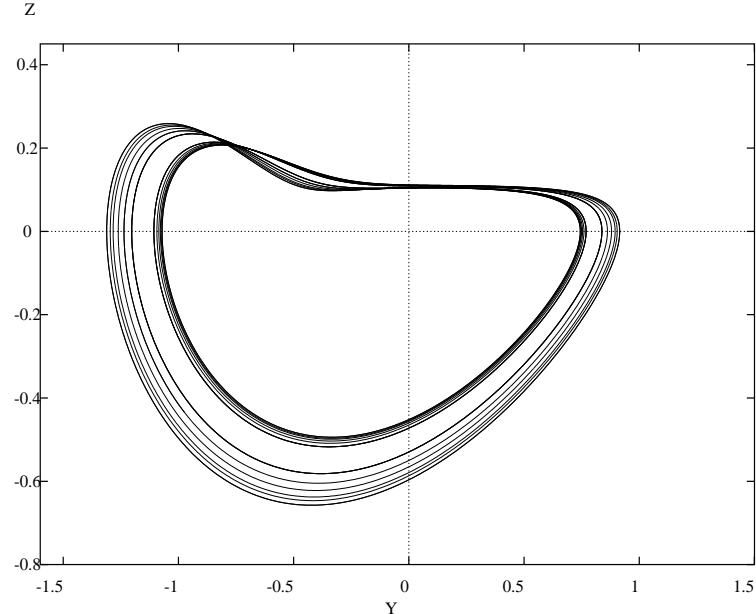

Figure 11: System (3.6b) period 12 solution. $A=-0.115, x(0)=0.3112473, y(0)=-0.973356$, $z(0)=-0.43474$

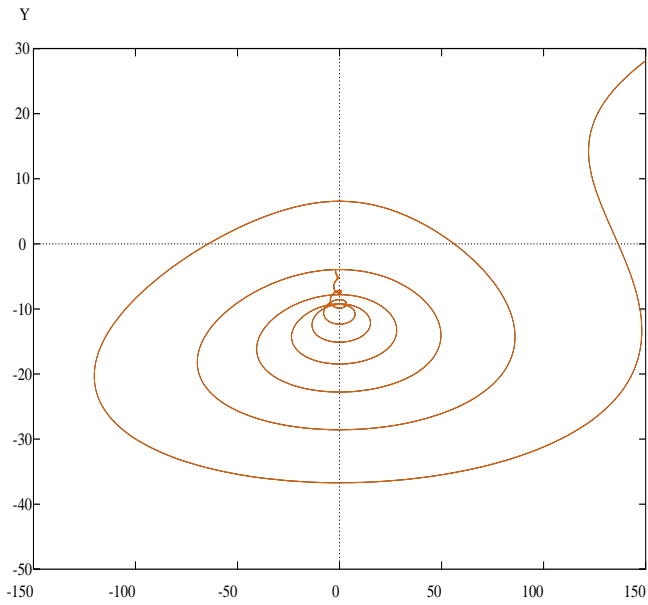

(a)

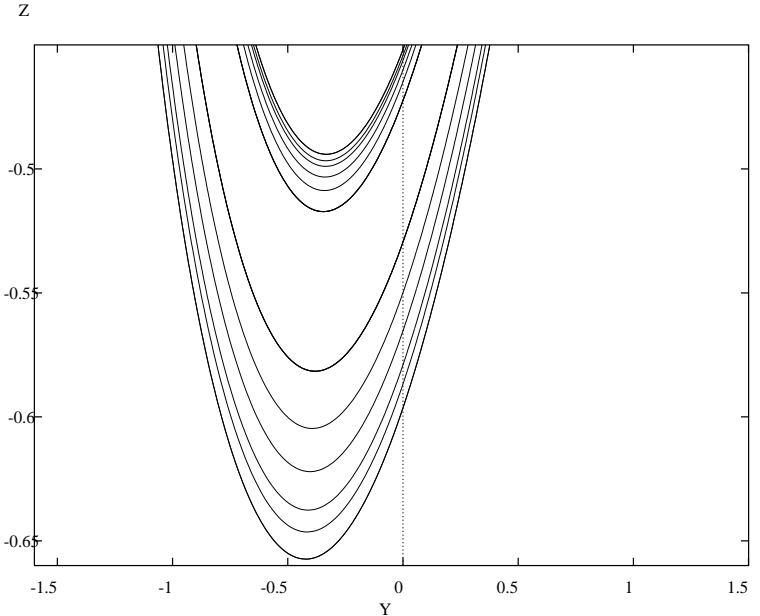

Figure 12: System (3.6b) period 12 solution. $A=-0.115, x(0)=0.3112473, y(0)=-0.973356$, $z(0)=-0.43474$

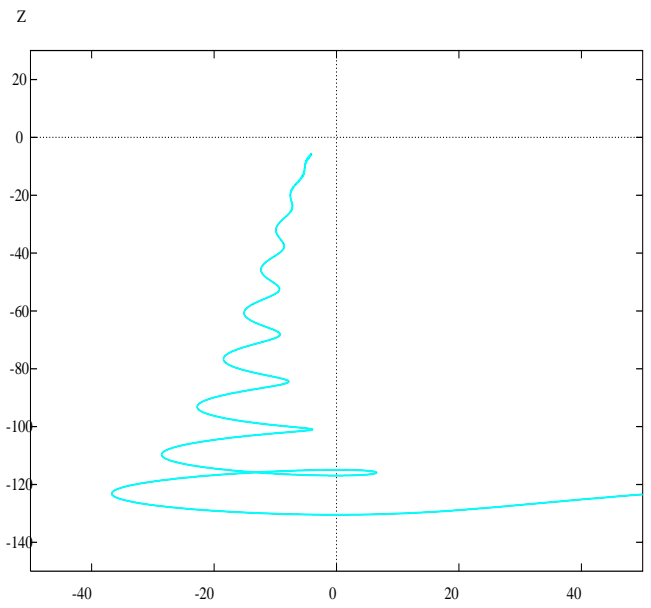

(b)

Figure 13: System (3.1a) picture (a) and (b) show that $x$ and $y$ oscillate several times and then become monotone, $z$ has only one turn before becoming monotone. $A=2.51, x(0)=-1.91, y(0)=-4.07, z(0)=-5.81$ 


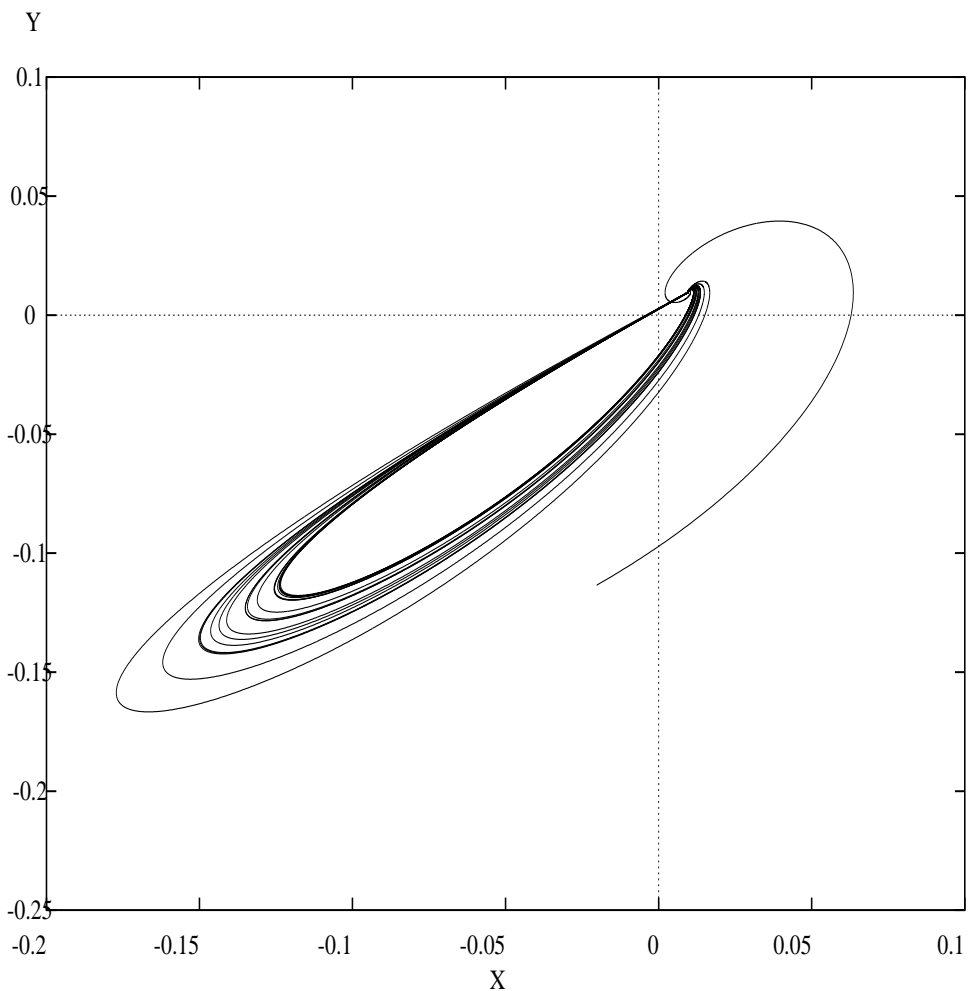

Figure 14: A strange attractor of system $(3.35 b)$

$A=-0.009453473, x(0)=-0.02022133354, y(0)=-0.11344168, z(0)=-1.030428604$ 


\section{Numerical Simulations}

We use Ermentrout's XPP [2] for our numerical simulations. The numerical method is fourth order RungaKutta (RK4), Relative Tolerence $=1 e-008$, Absolute Tolerence $=1 e-007$, and $\Delta t$ varies from 0.001 to 0.05. We used CVode for a couple of systems. We didn't find chaotic behavior in the systems studied in this section. In the following all the characteristic polynomials are for the linearization of the system at one of its equilibria.

The solutions of system (3.11a) with $A>-1$ oscillate and then become out of bound as shown in Figure 15 (a) and (b). When $x<0$, it is very easy to catch a solution that looks like the one shown in Figure 15. Other solutions are relatively simple according to our simulations. The equilibrium $(0,0,0)$ of the system has the characteristic polynomial $\lambda^{2}(1-\lambda)$, which is independent of $A$. The solutions of system (3.11b) with $A<1$ oscillate and then become unbounded as shown in Figure 16 and Figure 17. The equilibrium $(0,0,0)$ of the system has the characteristic polynomial $\lambda^{2}(1+\lambda)$, which is also independent of $A$. Since both systems have two zero eigenvalues, the behavior of them can be complicated.

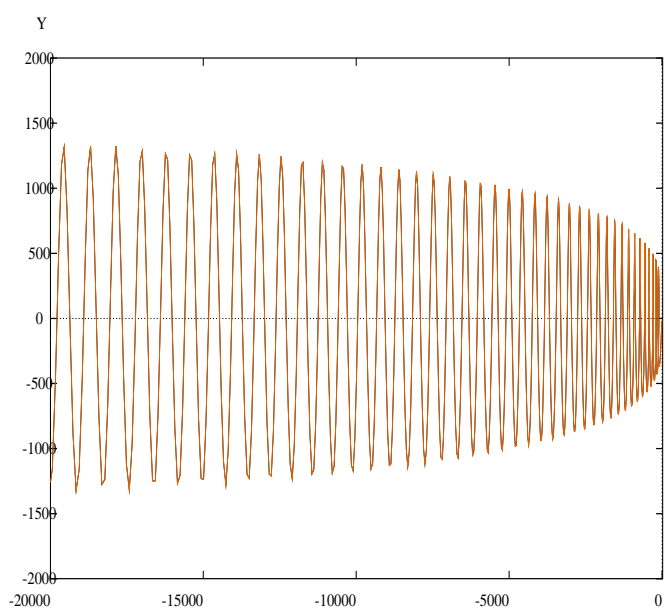

$(\mathrm{a})^{x}$

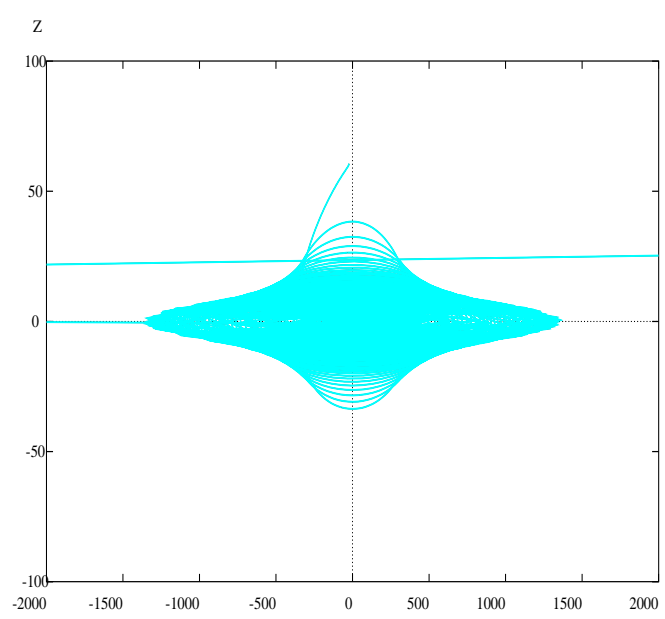

$(\mathrm{b})^{\mathrm{Y}}$

Figure 15: System (3.11a) $A=-0.992, x(0)=-1.095, y(0)=-22.47, z(0)=60.6$

For system (3.13a) and (3.13b) with $A<0$, from our simulations, typical solutions of system (3.13a) oscillate to become unbounded and typical solutions of system (3.13b) oscillate to approach the equilibrium $(0,0,0)$ as shown in Figure 18, Figure 19 and Figure 20. The characteristic polynomials of the equilibrium $(0,0,0)$ are $(-\lambda+1)\left(\lambda^{2}-A\right)$ and $(-\lambda-1)\left(\lambda^{2}-A\right)$ for the two systems and therefore the eigenvalues are +1 and -1 both with two imaginary roots $\pm \sqrt{A}$ respectively. Both systems should have bounded oscillatory solutions.

System (3.18a) and system (3.18b) both with $A<0$ have the same characteristic polynomials correspond to their equilibrium $(0,0,0)$ as those of $(3.13 a)$ and $(3.13 b)(\lambda-1)\left(\lambda^{2}-A\right)$ and $(\lambda+1)\left(\lambda^{2}-A\right)$ and therefore the eigenvalues are +1 and -1 both with two imaginary roots $\pm \sqrt{A}$ respectively. From our simulations, similar to the solutions of (3.13) the solutions of system (3.18a) oscillate to become unbounded and the solutions of system (3.18b) oscillate to approach the equilibrium as shown in Figure 21 and Figure 22 .

For system $(3.22 a)$ and $(3.22 b)$ both with $A<0$, the equilibrium $(0,0,0)$ have the characteristic poly- 


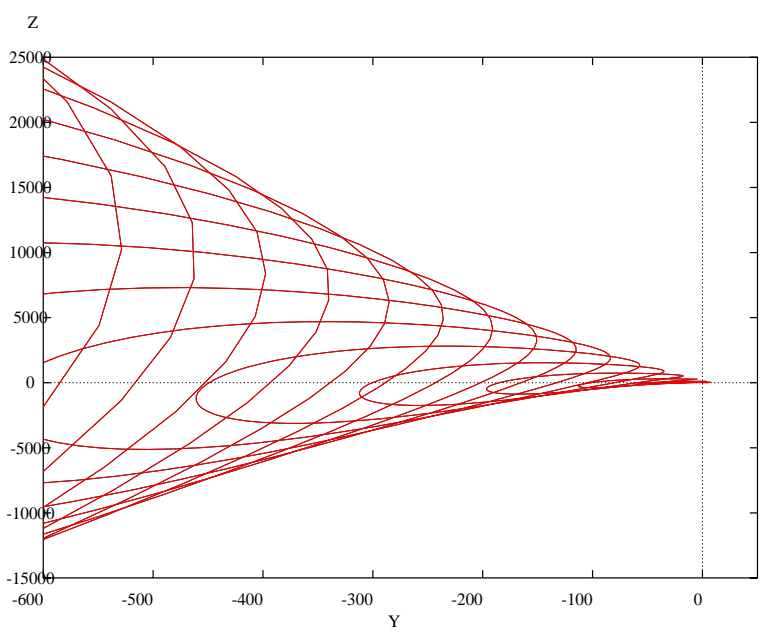

Figure 16: System (3.11b)

$A=-5.5, x(0)=-1.42, y(0)=12.64, z(0)=-4.01$

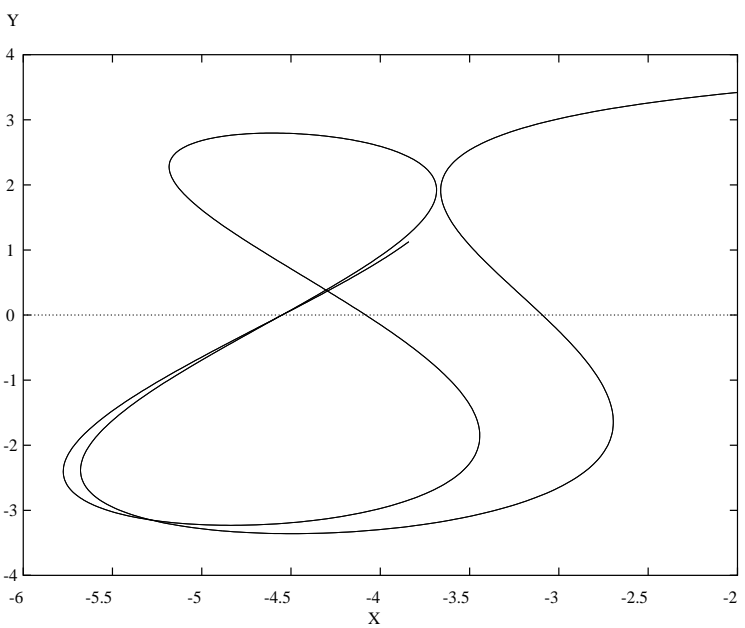

Figure 18: System (3.13a)

$A=-5.2, x(0)=-3.84, y(0)=1.128, z(0)=0.26$



Figure 17: System (3.11b)

$A=0.98, x(0)=-1.042, y(0)=283.7, z(0)=-233.7$

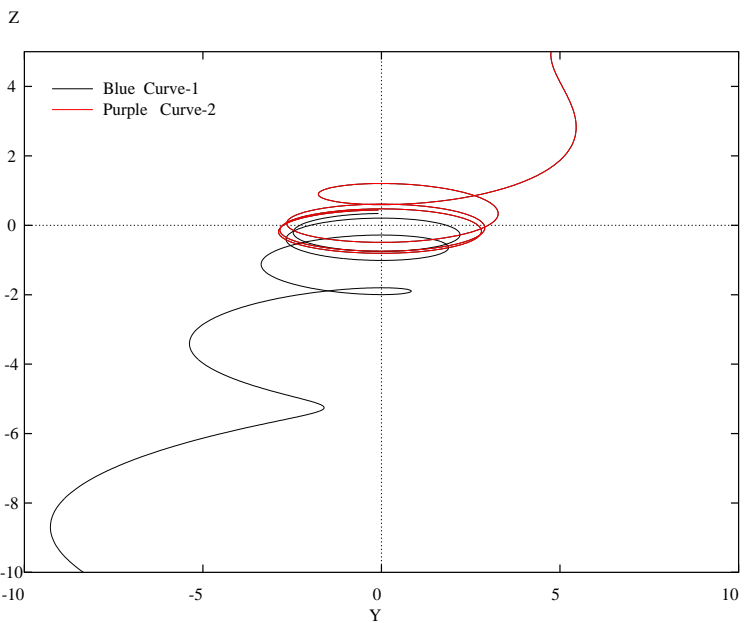

Figure 19: System (3.13a)

Curve-1: $A=-20.305, x(0)=-3.8, y(0)=-0.08$, $z(0)=0.339$; Curve-2 $A=-20.305, x(0)=-3.8$, $y(0)=-0.08, z(0)=0.439$ 


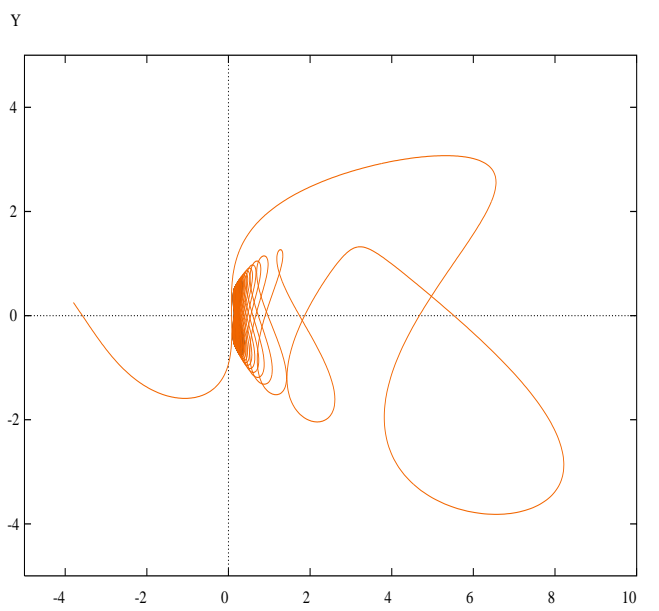

$(\mathrm{a})^{x}$

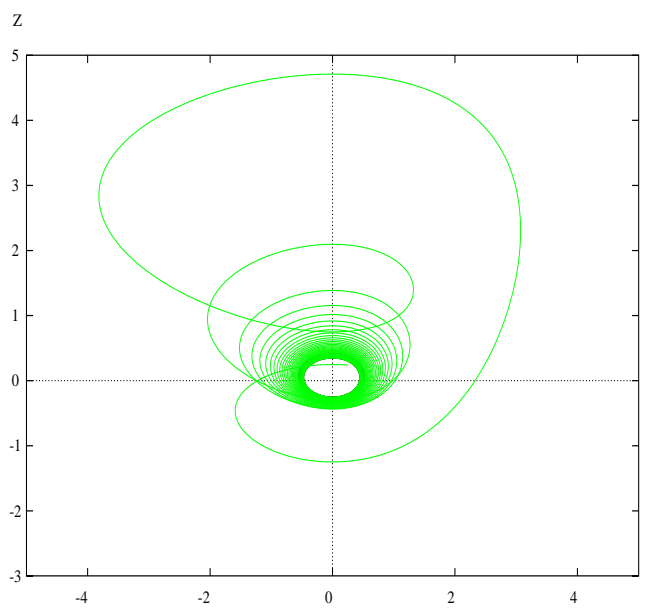

$(\mathrm{b})^{\mathrm{Y}}$

Figure 20: (a), (b) (3.13b) $A=-2.305, x(0)=-3.8, y(0)=0.252, z(0)=0.24$

nomials $( \pm 1-\lambda)\left(\lambda^{2}-A\right)$, which is the same as that of system (3.13). From our simulations, when $A$ is negative with large absolute value, curve 1 in Figure 23 oscillates around $x=0$ in the $x$ direction and $y$ becomes unbounded, curve 2 in Figure 23 also oscillates around $x=0$ in the $x$ direction and $y$ becomes unbounded. It seems that between curve 1 and curve 2 there is an unstable periodic solution. In Figure 24 both curve 1 and curve 2 seem to oscillate to either a stable periodic solution or the equilibrium.

For system (3.24a) and (3.24b) both with $A<0$, the linearization at the equilibrium $(0,0,0)$ have the characteristic polynomials $( \pm 1-\lambda)\left(\lambda^{2}-A\right)$, which is the same as that of system (3.13), and therefore the eigenvalues are +1 and -1 both with two imaginary roots $\pm \sqrt{A}$ respectively. Typical solutions of system (3.24a) oscillate and then move away from near the equilibrium and become unbounded as shown in Figure 25. Typical solutions of system (3.24b) become oscillatory and they approach either a stable periodic solution or the equilibrium as shown in Figure 26. The approach is very slow.

For system (3.31a) and (3.31b) both with $A<0$, the linearization at the equilibrium $(0,0,0)$ have the characteristic polynomials $( \pm 1-\lambda)\left(\lambda^{2}-A\right)$, which is the same as that of system (3.13), and therefore the eigenvalues are +1 and -1 both with two imaginary roots $\pm \sqrt{A}$ respectively. Typical solutions of system (3.31a) oscillate and then move away from the equilibrium and become unbounded as shown in Figure 27. Typical solutions of system (3.31b) become oscillatory and approach either a stable periodic solution or the equilibrium. For system $(3.31 b)$ we used CVode for the simulation.

For system (3.44a) with $A<1$ and system (3.44b) with $A>-1$, the equilibrium $(0,0,0)$ have characteristic polynomials $\lambda(\lambda-A)( \pm 1-\lambda)$ respectively. The solution shown in Figure 28 with positive small $A$ oscillate with the amplitude in $y$ direction increases and then decreases while the amplitude in $z$ direction increases to infinity. The solution of system (3.44b) shown in Figure 29 have similar behavior as that in Figure 28 in the sense that the $z$-amplitude becomes infinity. Picture (b) is the continuation of the solution in (a).

Now we look at system (4.6) with $A \in \mathbb{R}$. System (4.6a) and System (4.6b) both have two equilibria: $P_{1}^{ \pm}=(0,0,-A)$ and $P_{2}^{ \pm}=(\mp A, 0,0), P_{j}^{+}$and $P_{j}^{-}$are for " $a$ " and " $b$ " system respectively. The linearization at the equilibrium $P_{1}^{ \pm}=(0,0,-A)$ and $P_{2}^{ \pm}=(\mp A, 0,0)$ have the characteristic polynomials $-\lambda^{3} \pm \lambda^{2}-A$ and $-\lambda^{3}+\lambda^{2} \mp A \lambda \pm A$ respectively. Therefore the eigenvalues for the first equilibrium 


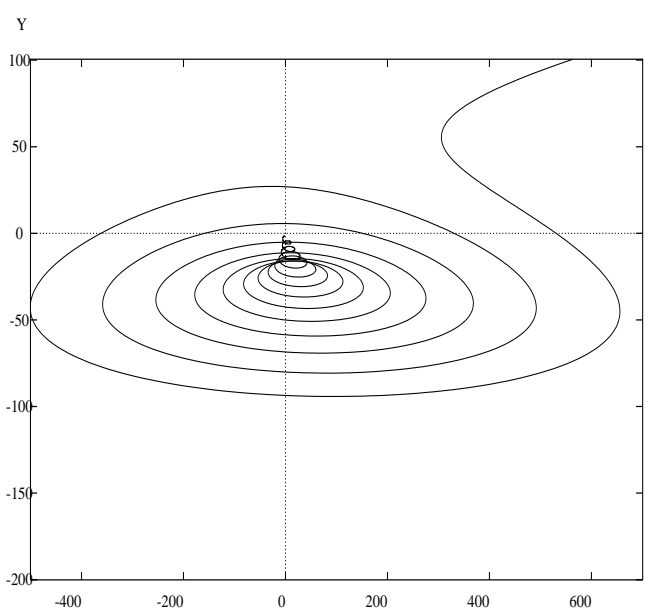

$($ a)

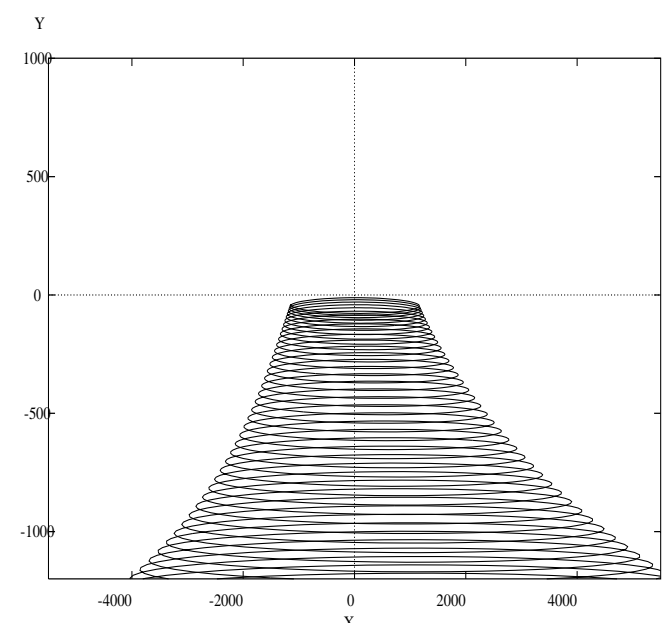

$(\mathrm{b})^{x}$

Figure 21: (a) System (3.18a) $A=-10.7, x(0)=-1.35, y(0)=-1.43, z(0)=2.18$

(b) System (3.18a) $A=-1000.7, x(0)=-1149.2, y(0)=-39.7, z(0)=2.18$

$P_{1}^{ \pm}=(0,0,-A)$ are the same as that of system (3.1) with two complex roots for certain $A$ s. The solutions shown in Figure 30 oscillate for small $t$ and then becomes unbounded. The solution shown in Figure 31 oscillates to approach the equilibrium or a stable periodic solution. System (4.6a) has an invariant region:

$$
\Omega_{1}=\{(x, y, z) \mid x+z+A \geqslant 0, y>0, z>0, A \leqslant 0\}
$$

Once a solution gets into this region it will go to infinity.

System (4.10a) and system (4.10b) both with $A<0$ have no fixed points. The solutions of system $(4.10 a)$ and $(4.10 b)$ shown in Figure 32 and Figure 33 started with oscillations and then become unbounded.

Finally we look at system (4.12a) with $A>0$ and system (4.12b) with $A<0$. System $(4.12 a)$ and system (4.12b) both have two equilibria: $P_{a}^{ \pm}=(0, \pm \sqrt{A}, \mp \sqrt{A})$ and $P_{b}^{ \pm}=(0, \pm \sqrt{-A}, \pm \sqrt{-A}), P_{a}^{ \pm}$and $P_{b}^{ \pm}$are for " $a$ " and " $b$ " system respectively. The linearization at the equilibrium $P_{a}^{ \pm}$and $P_{b}^{ \pm}$have the characteristic polynomials $-\lambda^{3}+\lambda^{2} \pm \sqrt{A} \lambda \mp 2 \sqrt{A}$ and $-\lambda^{3}-\lambda^{2} \pm \sqrt{-A} \lambda \pm 2 \sqrt{-A}$. The solutions of system (4.12a) and (4.12b) shown in Figure $34(a)$ and Figure $34(b)$ again started with oscillations and then become unbounded.

Our simulations suggest that it is unlikely that systems (3.11), (3.44) and (4.10) have chaos. 




Figure 22: System $(3.18 b)$

Curve-1: $A=-1.117, x(0)=0.63, y(0)=-0.88, z(0)=-0.28$

Curve-2: $A=-1.117, x(0)=-0.38, y(0)=1.27, z(0)=-0.28$

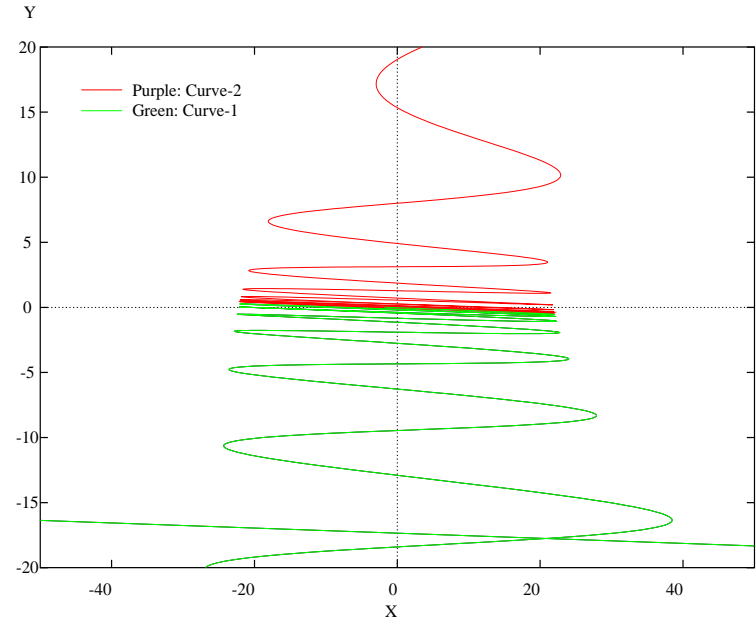

Figure 23: System $(3.22 a)$



Figure 24: System $(3.22 b)$

Curve-1: $A=-50, x(0)=-1.8, y(0)=0.01, z(0)=-3.1$ Curve-1: $A=-100.7, x(0)=1.6, y(0)=-70, z(0)=0.5$ Curve-2: $A=-50, x(0)=-1.8, y(0)=0.1, z(0)=-3.1 \quad$ Curve-2: $A=-100.7, x(0)=1.6, y(0)=70, z(0)=247$ 




$(\mathrm{a})^{\mathrm{x}}$

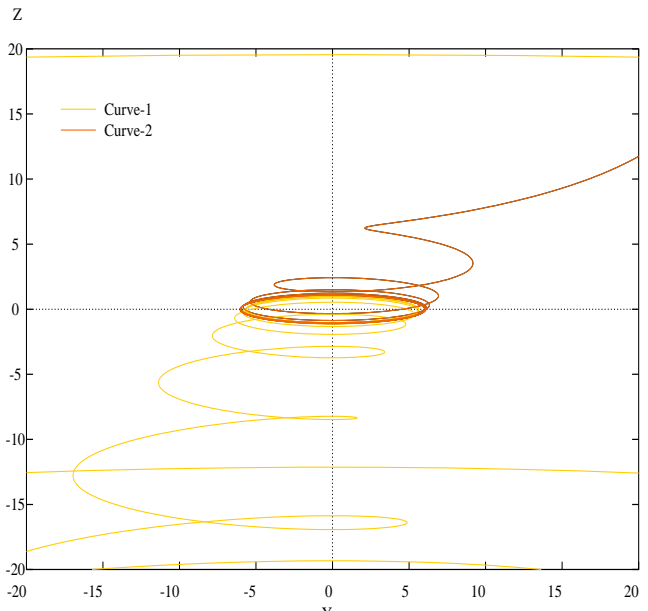

$(\mathrm{b})^{x}$

Figure 25: (a) System (3.24a) Curve-1: $A=-20.55, x(0)=-0.59, y(0)=1.915, z(0)=0.9396$. Curve-2: $A=$ $-20.55, x(0)=-0.6, y(0)=1.915, z(0)=0.9396$

(b) System (3.24a) Curve-1: $A=-30.55, x(0)=-0.6, y(0)=1.915, z(0)=0.9396$. Curve-2: $A=-30.55, x(0)=-0.6, y(0)=2.915, z(0)=0.9396$

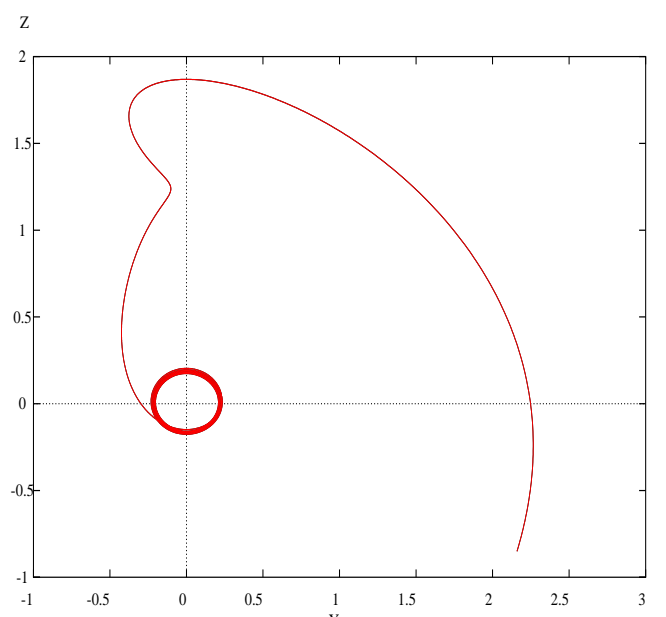

$(\mathrm{a})^{Y}$

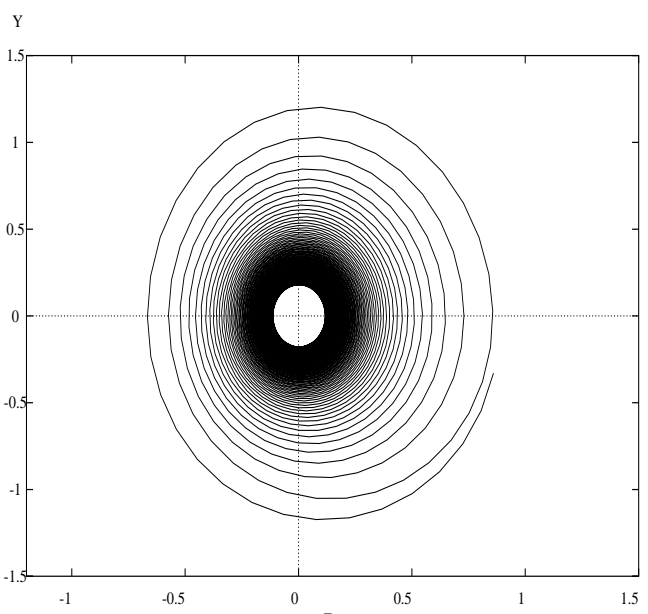

$(b)^{2}$

Figure 26: (a) System (3.24b) $A=-0.512, x(0)=-0.2, y(0)=-0.46, z(0)=1.37583$ (b) System (3.24b) $A=-2.41, x(0)=0.25, y(0)=-0.33, z(0)=0.86$ 


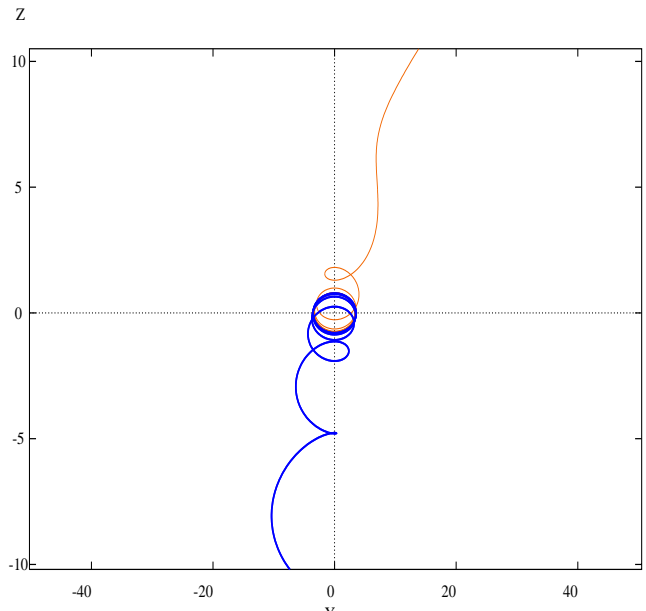

$(\mathrm{a})^{Y}$

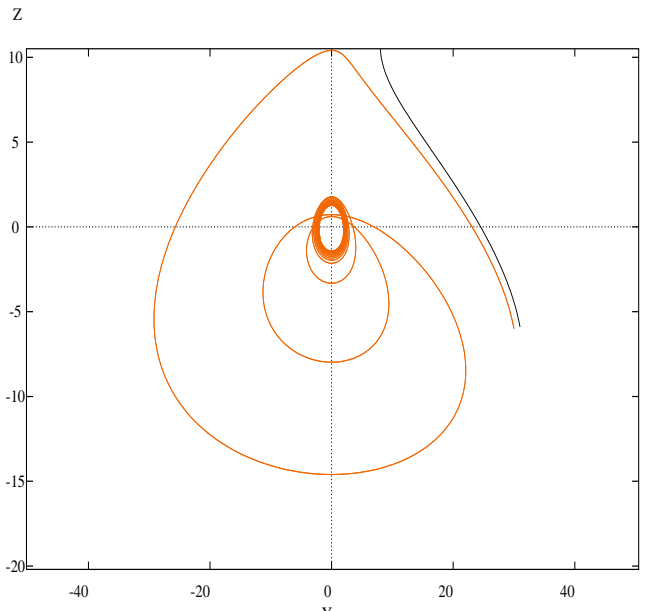

$(b)^{y}$

Figure 27: (a) System (3.31a) $A=-20, x(0)=0, y(0)=-2.5, z(0)=0.5$ (red), $z(0)=0.55$ (blue)

(b) System (3.31b) $A=-2.2, x(0)=-29, y(0)=30, z(0)=-6$ (red), $y(0)=31, z(0)=-5.9$ (blue)

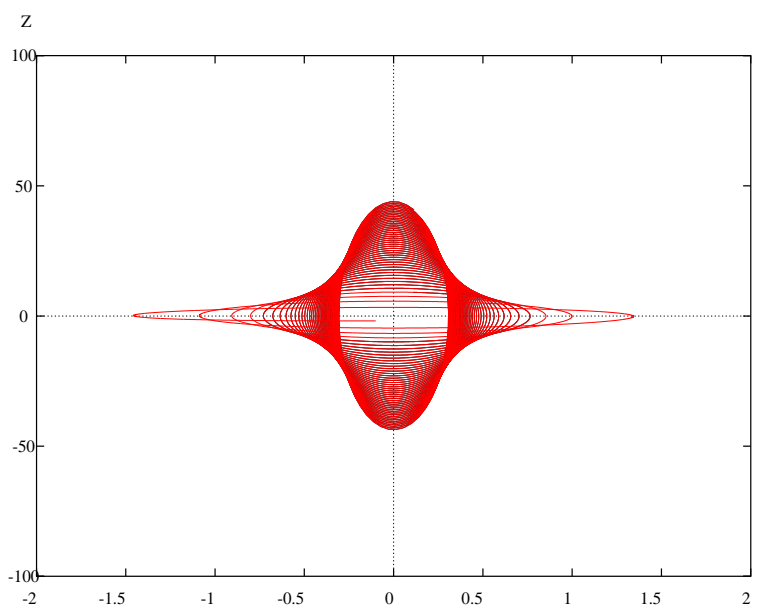

$(\mathrm{a})^{\mathrm{Y}}$

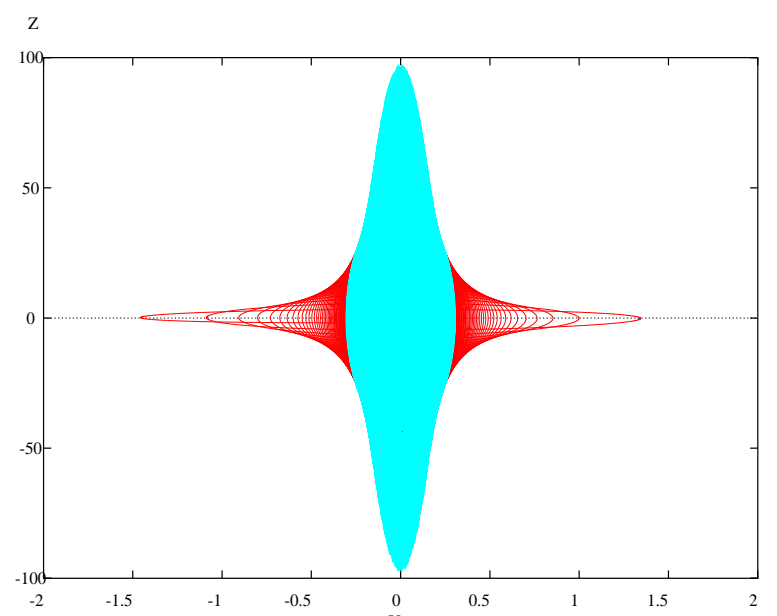

(b)

Figure 28: System (3.44a)

(a) $A=0.125, x(0)=-0.5, y(0)=-0.1, z(0)=-1.9$

(b) Same parameter and initial condition with longer time 


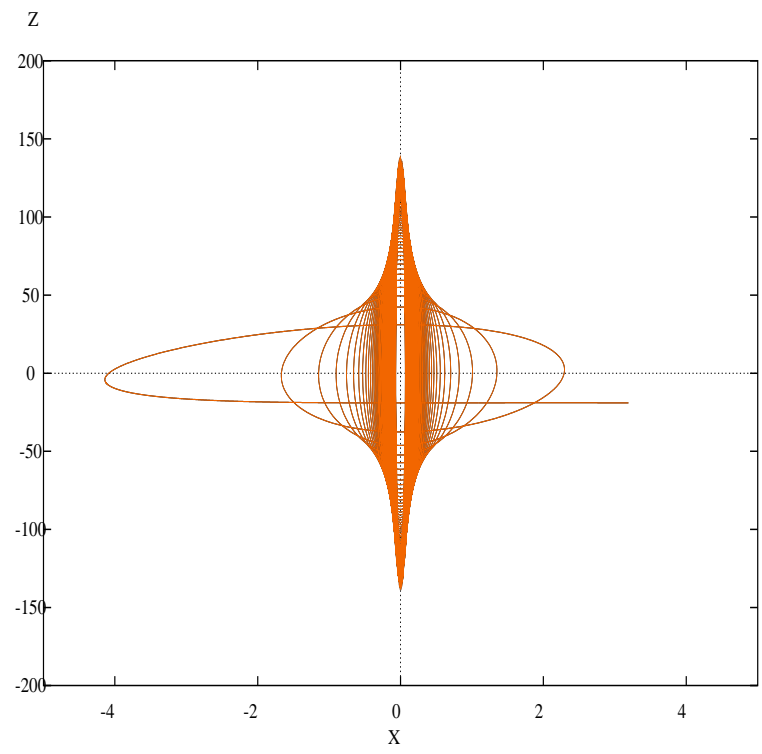

Figure 29: System $(3.44 b)$

$A=5.65, x(0)=3.19, y(0)=0.8, z(0)=-19$

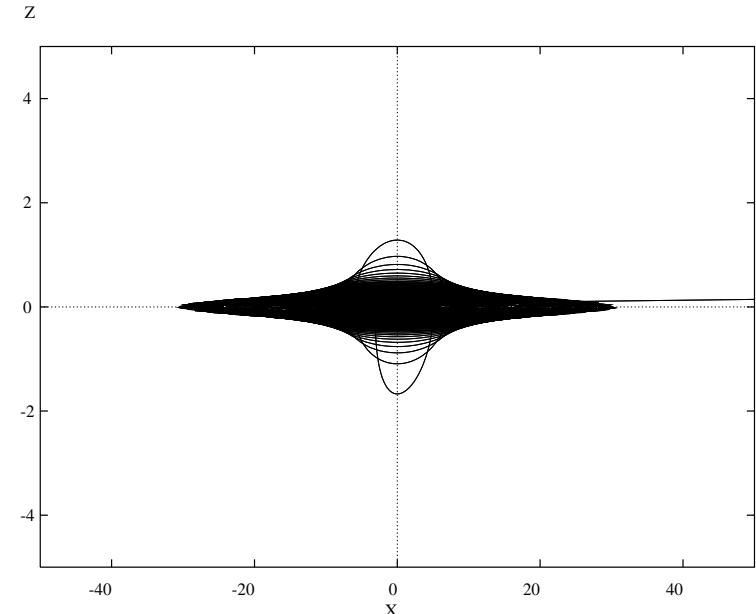

Figure 30: System (4.6a)

$A=-1.65, x(0)=-1.2, y(0)=3.1, z(0)=-0.4$

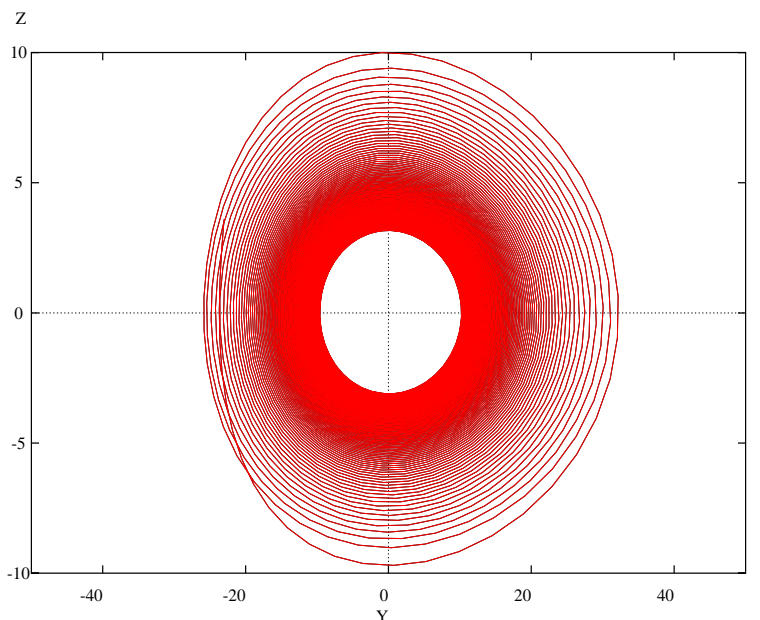

Figure 31: System (4.6b) $A=-10.05, x(0)=-2.2, y(0)=-23, z(0)=3.6$ 


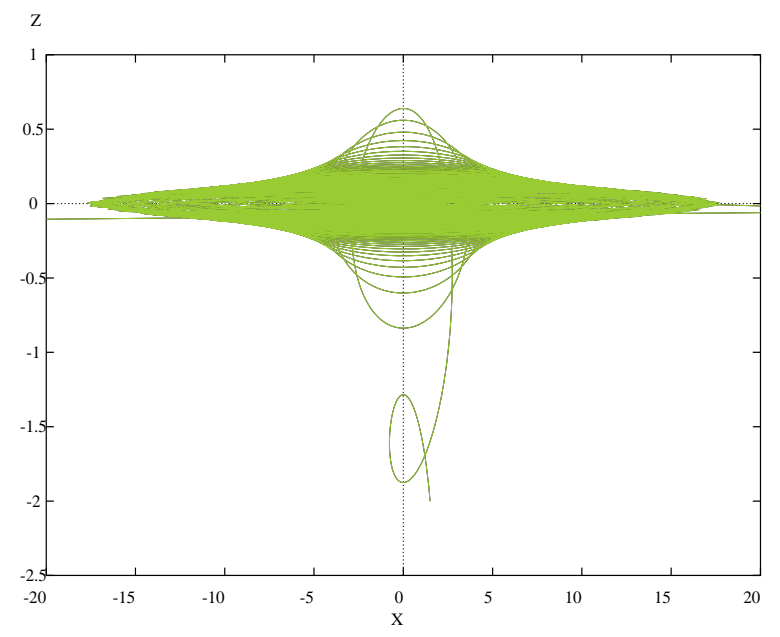

Figure 32: System $(4.10 a)$

$A=-2.65, x(0)=1.5, y(0)=-0.8, z(0)=-2$

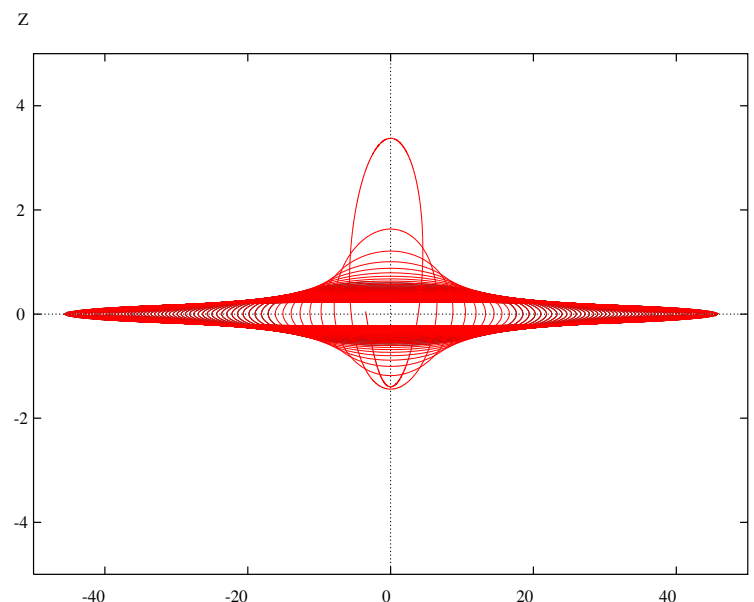

(a)

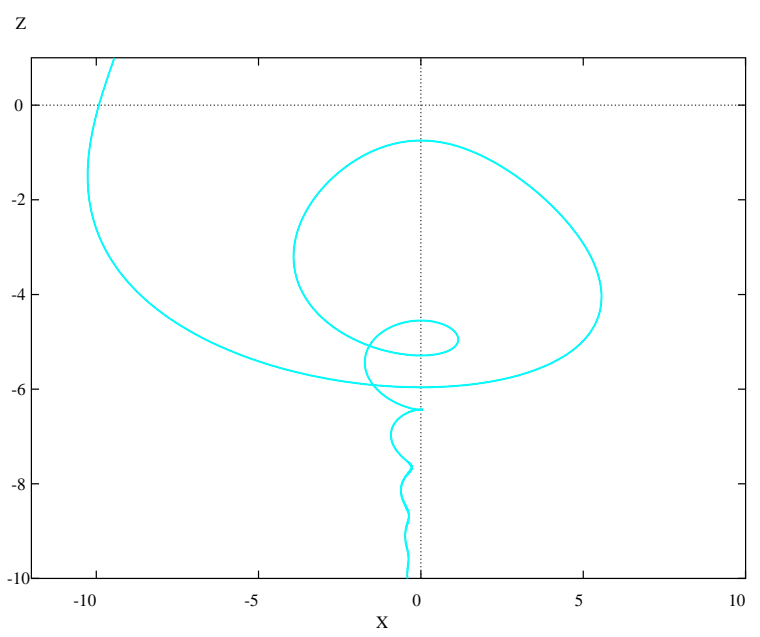

Figure 33: System $(4.10 b)$

$A=-4.125, x(0)=-8.8, y(0)=0.8, z(0)=2.5$

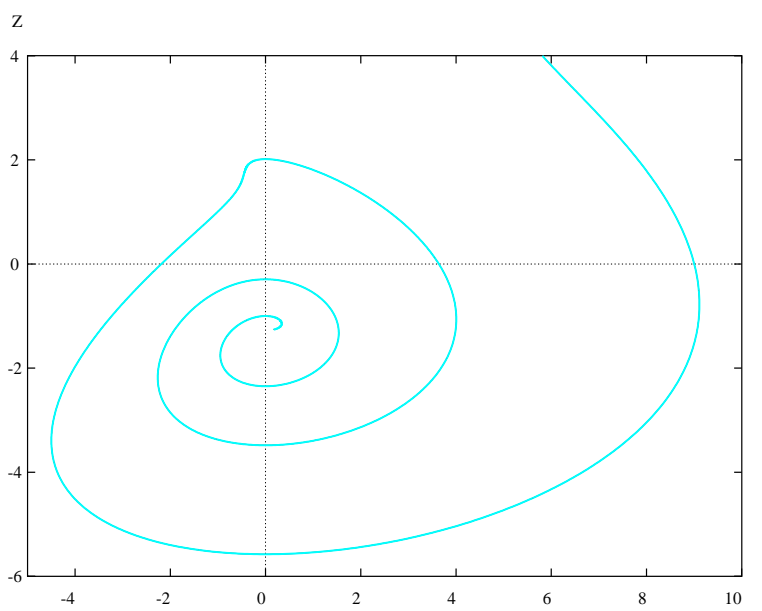

(b) ${ }^{\mathrm{x}}$

Figure 34: System (4.12)

(a) (4.12a) $A=4.125, x(0)=-3.5, y(0)=0.08, z(0)=0.05$

(b) $(4.12 b) A=-2.5, x(0)=0.17, y(0)=-2.67, z(0)=-1.26$ 


\section{Conclusion}

In the first part of this paper we proposed and proved an extension of Theorem 2.1 in [6]. The difference between Theorem 2.3 and Theorem 2.1 in [6] is in their hypotheses. Theorem 2.3 allows $f(x)$ to be continuous or to have singularities. None of them implies the other. There are systems that can be resolved by both theorems. There are also systems that can be resolved by one but not the other. To apply the new theorem, one often need more calculations to verify $(H 2)$.

We then considered all possible 5-1 quadratic dissipative systems. Out of all possible 5-1 quadratic dissipative systems we obtained 65 systems that need to be further studied. We proved analytically that 44 of the 65 systems are nonchaotic. Nine out of the 21 chaotic candidates are found to exhibit chaos. But one only has to study two of them, $(3.1 b)$ and $(3.35 b)$ because the remaining seven systems are equivalent to one of the two. It is worth mentioning that chaotic solutions in system (3.35b) are very difficult to find. For the remaining 12 systems we didn't find chaotic solutions after an extensive numerical simulations. We believe that there is no chaos in systems (3.11), (3.44) and (4.10).

Finally we point out that in [8] (Malasoma, 2002) the systems have the following equivalent relations: for the systems in Malasoma's paper we denote them by $\mathrm{M}(n), n=5,6,7,8,11,16,18$.

$$
\begin{aligned}
& M(5)\left\{\begin{array}{l}
x^{\prime}=y+1 \\
y^{\prime}=-\alpha y+z \quad \text { exchange } x \text { and } y, \quad \text { Z-H(4.20) }\left\{\begin{array}{l}
x^{\prime}=-\alpha x+z \\
y^{\prime}=x+1 \\
z^{\prime}=x y
\end{array} \quad x y\right.
\end{array}\right. \\
& M(6)\left\{\begin{array}{l}
x^{\prime}=z \\
y^{\prime}=x+1 \\
z^{\prime}=-\alpha z+x y
\end{array} \quad \text { exchange } x \text { and } z, \quad \mathrm{Z}-\mathrm{H}(4.17)\left\{\begin{array}{l}
x^{\prime}=-\alpha x+y z \\
y^{\prime}=z+1 \\
z^{\prime}=x
\end{array}\right.\right. \\
& M(7)\left\{\begin{array}{l}
x^{\prime}=y \\
y^{\prime}=-\alpha y+z \\
z^{\prime}=-x+y^{2}
\end{array} \quad \text { exchange } x \text { and } z, \quad \mathrm{Z}-\mathrm{H}(3.19)\left\{\begin{array}{l}
x^{\prime}=-z+y^{2} \\
y^{\prime}=-\alpha y+x \\
z^{\prime}=y
\end{array}\right.\right. \\
& M(8)\left\{\begin{array}{l}
x^{\prime}=y \\
y^{\prime}=-\alpha y+z \\
z^{\prime}=-x+x y
\end{array} \quad \text { exchange } x \text { and } z, \quad \mathrm{Z}-\mathrm{H}(3.36)\left\{\begin{array}{l}
x^{\prime}=-z+z y \\
y^{\prime}=-\alpha y+x \\
z^{\prime}=y
\end{array}\right.\right. \\
& M(11)\left\{\begin{array} { l } 
{ x ^ { \prime } = z } \\
{ y ^ { \prime } = - \alpha y + z \quad \text { exchange } x \text { and } z , \quad \text { Z-H } ( 3 . 3 5 ) } \\
{ z ^ { \prime } = - x + x y }
\end{array} \left\{\begin{array}{l}
x^{\prime}=-z+z y \\
y^{\prime}=-\alpha y+x \\
z^{\prime}=x
\end{array}\right.\right.
\end{aligned}
$$

$M(16)\left\{\begin{array}{l}x^{\prime}=z \\ y^{\prime}=-\beta y+z-1 \\ z^{\prime}=x y\end{array} \quad\right.$ exchange $x$ and $y\left\{\begin{array}{l}x^{\prime}=-\beta x+z-1 \\ y^{\prime}=z \\ z^{\prime}=x y\end{array} \quad\right.$ then $y$ and $z,\left\{\begin{array}{l}x^{\prime}=-\beta x+y-1 \\ y^{\prime}=x z \\ z^{\prime}=y\end{array}\right.$

where the last one is $\mathrm{Z}-\mathrm{H}(4.5)$

$$
M(18)\left\{\begin{array} { l } 
{ x ^ { \prime } = z + 1 } \\
{ y ^ { \prime } = - \beta y + z } \\
{ z ^ { \prime } = x y }
\end{array} \quad \text { exchange } x \text { and } y \left\{\begin{array}{l}
x^{\prime}=-\beta x+z \\
y^{\prime}=z+1 \\
z^{\prime}=x y
\end{array} \quad \text { then } y \text { and } z,\left\{\begin{array}{l}
x^{\prime}=-\beta x+y \\
y^{\prime}=x z \\
z^{\prime}=y+1
\end{array}\right.\right.\right.
$$

Then let $Y=y+1$. M(18) becomes Z-H(4.5)

$$
\left\{\begin{array}{l}
x^{\prime}=-\beta x+Y-1 \\
Y^{\prime}=x z \\
z^{\prime}=Y
\end{array}\right.
$$


Acknowledgement The authors thank the referees for their valuable suggestions.

\section{References}

[1] Alligood, K. T., Sauer, T. D., and Yorke, J. A., CHAOS: An introduction to Dynamical Systems, (Springer-Verlag), 1996

[2] Ermentrout, B., XPP ordinary differential equation and difference equation integrators

[3] Frederickson, P, Kaplan, J. L. , Yorke, E. D., and Yorke, J. A., The Liapunov dimension of strange attractors, Original Research Article Journal of Differential Equations, Volume 49, Issue 2, August 1983, Pages 185-207

[4] Hartman, Philip, Ordinary Differential Equations. John Wiley and Sons, Inc. N.Y., 1964.

[5] Heidel, J. and Zhang F, Nonchaotic Behaviour in the Three-Dimensional Quadratic Systems II: The conservative case, Nonlinearity,1999, No.12 617-633

[6] Heidel, J. and Zhang F, Nonchaotic and Chaotic Behavior in Three-Dimensional Quadratic Systems: Five-One Conservative Cases, International Journal of Bifurcation and Chaos, Vol. 17, No.6(2007) 2049-2072.

[7] Lorenz, N. E, Deterministic non-periodic flow. J. Atmos Sci. 1963,No.20 130

[8] Malasoma, J. -M, A new class of minmal chaotic flowsequation for continuous chaos. Phys. Lett. A $305(2002) 52-58$

[9] Rössler, J. C, An equation for continuous chaos. Phys. Lett. A 1976 vol.57: 397.

[10] Sprott, J. C, Some simple chaotic flow. Phys. Rev. E Vol 50, No. 2 (1994), R647-R650

[11] Sprott, J. C, Some simple chaotic jerk functions. Am. J. Phys. 65(1997), 537-543

[12] Sprott, J. C, Simplest dissipative chaotic flow. Phys. Lett. A 228(1997), 271-274

[13] Sprott, J. C, Algebraically Simple chaotic flows. International Journal of Chaos Theory and Applications, Vol 5 (2000), No. 2

[14] Zhang, F. and Heidel, J, Nonchaotic Behaviour in the Three-Dimensional Quadratic Systems, Nonlinearity, 1997, No.10 